\title{
A Class of Evolutionary Models for Participation Games with Negative Feedback
}

\author{
Pietro Dindo · Jan Tuinstra
}

Accepted: 3 January 2011 / Published online: 3 February 2011

(C) The Author(s) 2011. This article is published with open access at Springerlink.com

\begin{abstract}
We introduce a framework to analyze the interaction of boundedly rational heterogeneous agents repeatedly playing a participation game with negative feedback. We assume that agents use different behavioral rules prescribing how to play the game conditionally on the outcome of previous rounds. We update the fraction of the population using each rule by means of a general class of evolutionary dynamics based on imitation, which contains both replicator and logit dynamics. Our model is analyzed by a combination of formal analysis and numerical simulations and is able to replicate results from the experimental and computational literature on these types of games. In particular, irrespective of the specific evolutionary dynamics and of the exact behavioral rules used, the dynamics of the aggregate participation rate is consistent with the symmetric mixed strategy Nash equilibrium, whereas individual behavior clearly departs from it. Moreover, as the number of players or speed of adjustment increase the evolutionary dynamics typically becomes unstable and leads to endogenous fluctuations around the steady state. These fluctuations are robust with respect to behavioral rules that try to exploit them.
\end{abstract}

Previous versions of this paper have circulated under the title "A behavioral model for participation games with negative feedback".

P. Dindo $(\varangle)$

LEM, Scuola Superiore Sant’Anna, Piazza Martiri della Libertà 33, 56127 Pisa, Italy

e-mail: p.dindo@sssup.it

J. Tuinstra

Department of Quantitative Economics and CeNDEF, Faculty of Economics and Business,

University of Amsterdam, Amsterdam, The Netherlands

e-mail: J.Tuinstra@uva.nl 
Keywords Participation games - Heterogeneous behavioral rules - Revision protocol $\cdot$ Replicator dynamics $\cdot$ Logit dynamics · Nonlinear dynamics

\section{JEL Classification $\mathrm{C} 72 \cdot \mathrm{C} 73$}

\section{Introduction}

Many economic decisions, such as firms choosing whether or not to enter a new market or to invest in a new technology, commuters choosing a route to the workplace, or suppliers choosing between two locations to sell their products, can be characterized as participation games with negative feedback. This type of game is encountered whenever a, possibly large, group of agents is competing for a scarce resource in the absence of a market institution that acts as a coordination device. Their strategic setting can be exemplified as choosing whether or not to participate in a certain 'project', given that the payoff associated with participating decreases with the number of other players taking the same decision. In the terminology of Haltiwanger and Waldman (1985), actions in negative feedback participation games are strategic substitutes. ${ }^{1}$

In this paper we study the interaction of a group of boundedly rational heterogeneous agents who are repeatedly playing a participation game with negative feedback. Drawing from the experimental and computational literature we build a model where agents decide whether to participate or not using behavioral rules that condition on the outcome of previous rounds, as in Arthur (1994) and Selten et al. (2007). Moreover, through an evolutionary process, more successful rules "attract" a larger share of the population. Such an evolutionary competition between different, possibly sophisticated, behavioral rules, has been successfully introduced in a number of economic environments, such as cobweb models, financial markets, Cournot oligopoly models, and labor markets (see e.g., Brock and Hommes 1997, 1998; Chiarella and He 2002; Droste et al. 2002; Neugart and Tuinstra 2003 and Brianzoni et al. 2010) but is new to participation games.

The existing experimental literature on participation games suggests that when agents play them repeatedly the dynamics of the aggregate outcome is inherently unstable and the participation rate exhibits persistent fluctuations. At the aggregate level, the first moment of the empirical distribution of participation rates seems to be consistent with the symmetric mixed strategy Nash equilibrium of the underlying one-shot game. Nevertheless, individual subjects do not play according to this strategy. Participation rate fluctuations therefore cannot be easily attributed to the randomization implied by the mixed strategy Nash equilibrium. Subjects seem to base their decisions on different behavioral rules or heuristics. Existing models that have tried to replicate this feature suffer from two drawbacks: they are typically purely computational, and they rely on the choice of specific behavioral rules and/or updating dynamics so that the generality of the findings is questionable.

\footnotetext{
${ }^{1}$ In contrast, public good provision, union membership, or technology adoption may be characterized as participation games with positive feedback, that is, games where the payoff for participating increases with the number of participating players. Actions are in this case strategic complements. See Anderson and Engers (2007) for a similar definition of participation games.
} 
The aim of the present paper is to put forward a simple framework able to replicate the main stylized facts emerging from the experimental and computational results using a general class of evolutionary updating mechanisms and a general class of behavioral rules. Following recent work by Hofbauer and Sandholm (2009), the class of updating mechanisms is characterized by a number of properties of agents' propensities to switch between rules. It contains some well-known evolutionary models, such as the replicator dynamics and the logit dynamics. The class of behavioral rules is characterized by two stylized properties: agents condition their choices on past participation rates, and agents do not randomize.

We investigate under which conditions such an evolutionary dynamics converges, and if so, whether it converges to a Nash equilibrium of the participation game. In its most stylized form, when only simple behavioral rules are used, our model is low dimensional and analytically tractable. Both properties are lost, however, when more complicated rules are available, but insight into the evolutionary dynamics may then be gathered from numerical simulations.

Our first finding is that the participation rate may be unstable and fluctuates around the symmetric mixed strategy Nash equilibrium, even if individual behavior clearly departs from it. This finding is consistent with earlier results from the experimental and computational literature. Secondly, we show that the introduction of new rules that try to exploit a regularity in the time series of participation rates does not stabilize participation. In fact, adding new rules make the resulting time series more irregular and more unpredictable. The endogenous fluctuations found in this type of framework are therefore quite robust.

The remainder of the paper is organized as follows. In Sect. 2 the one-shot negative feedback participation game is introduced and its Nash-equilibria are characterized. We also briefly discuss the experimental and computational literature on participation games in that section. Section 3 introduces a framework for studying evolutionary competition between different rules and in Sect. 4 we apply this to a stylized, but very instructive and analytically tractable, example. In Sect. 5 we use simulations to investigate how the overall dynamics changes when more complex rules are added and, in particular, whether new rules can exploit the emerging patterns of past participation rates. Section 6 concludes. The appendix contains proofs of the main results.

\section{The Negative Feedback Participation Game}

We consider a participation game with $N$ players. Each player chooses an action $a \in\{0,1\}$, where $a=1$ represents 'participating' and $a=0$ represents 'not participating'. The action space is given by $A=\{0,1\}^{N}$ and an action profile by $\mathbf{a} \in A$. By $\mathbf{a}_{-i}=\left(a_{1}, \ldots, a_{i-1}, a_{i+1}, \ldots, a_{N}\right)$ we denote the set of actions played by all players but player $i$. The payoff $\pi_{i}\left(a_{i}, \mathbf{a}_{-i} ; N_{c}, N\right)$ is given as:

$$
\begin{aligned}
& \pi_{i}\left(0, \mathbf{a}_{-i} ; N_{c}, N\right)=\alpha, \\
& \pi_{i}\left(1, \mathbf{a}_{-i} ; N_{c}, N\right)=\left\{\begin{array}{ll}
\alpha+\beta-\gamma & \text { if } \sum_{j=1, j \neq i}^{N} a_{j}<N_{c} \\
\alpha-\gamma & \text { if } \sum_{j=1, j \neq i}^{N} a_{j} \geq N_{c}
\end{array},\right.
\end{aligned}
$$


where the parameter $N_{c}$ denotes the capacity of the project. Participating gives payoff $\alpha-\gamma$ if $N_{c}$ or more of the other $N-1$ players participate, and payoff $\alpha+\beta-\gamma$ if less than $N_{c}$ of the other $N-1$ players participate. ${ }^{2}$ We assume $\alpha>\gamma$ to ensure that payoffs are always strictly positive and $\beta>\gamma$ to ensure that $a=1$ is not a dominated strategy. The payoff $\alpha$ corresponds to some outside payment, ${ }^{3} \gamma$ to the cost or effort of participation and $\beta$ to the return of a successful project. Note that this is indeed a negative feedback participation game, since an increase in the number of players participating in the project might decrease profitability of the project.

In many experiments on participation games with negative feedback, e.g., market entry games, the payoff for participating is linearly decreasing in the number of other entrants. The present formulation, using a step function for the participating payoff, follows the payoff function for the El Farol bar game that was used in Arthur (1994), Bell (2001) and Franke (2003). We chose this stylized version of the game since it facilitates analyzing the effect of $N$ on the dynamics, while keeping the total number of parameters to a minimum.

A (mixed or pure) strategy $s_{i}$ for player $i$ is the probability with which he $\backslash$ she chooses action $a=1$. The strategy space is therefore given by $S=[0,1]^{N}, \mathbf{s} \in S$ denotes a strategy profile and $\mathbf{s}_{-i}$ denotes the set of strategies for all players, except player $i$. Using (1) the expected payoff of playing strategy $s_{i}$ is given by

$$
\begin{aligned}
\pi_{i}\left(s_{i}, \mathbf{s}_{-i} ; N_{c}, N\right) & =\left(1-s_{i}\right) \alpha+s_{i}\left(\alpha+\beta \operatorname{Pr}\left\{N_{-i} \leq N_{c}-1\right\}-\gamma\right) \\
& =\alpha+s_{i}\left(\beta \operatorname{Pr}\left\{N_{-i} \leq N_{c}-1\right\}-\gamma\right)
\end{aligned}
$$

where $\operatorname{Pr}\left\{N_{-i} \leq N_{c}-1\right\}$ is the probability that the number of other agents participating, $N_{-i}$, which is a random variable, is strictly smaller than $N_{c}$. Obviously, this probability depends upon the strategy profile $\mathbf{s}_{-i}$.

\subsection{Nash Equilibria}

Assume that all players are risk neutral and want to maximize their expected payoffs. The game has many pure strategy Nash equilibria (henceforth PSNE). Any strategy profile $\mathbf{s}$ such that exactly $N_{c}$ players participate with certainty $(s=1)$ and the other $N-N_{c}$ participants abstain with certainty $(s=0)$ corresponds to a strict PSNE. Evidently, there are $\left(\begin{array}{c}N \\ N_{c}\end{array}\right)$ of these PSNE. Note that such a PSNE leads to an uneven distribution of payoffs, with exactly $N_{c}$ players obtaining $\alpha+\beta-\gamma$ and the other $N-N_{c}$ players receiving $\alpha$. Now consider mixed strategy Nash equilibria (henceforth MSNE), where some players randomize between the two possible actions. We will prove that there exists a unique symmetric mixed strategy Nash equilibrium $s^{*} \in(0,1)$. If each

\footnotetext{
${ }^{2}$ Note that for $N=2$ and $N_{c}=1$, this payoff structure is similar to the well-known Hawk-Dove game (see e.g., Fudenberg and Tirole 1991, pp. 18-19)

${ }^{3}$ In this formulation of the game, the payoff $\alpha$ could be set to zero without changing the results. Nevertheless, since in the dynamic approach of the following sections $\alpha$ does play an important role, we have decided to introduce it at this earlier stage.
} 
player participates with probability $s^{*}$, the probability that strictly less than $N_{c}$ out of $N-1$ players participate is given by:

$$
p\left(s^{*} ; N_{c}, N\right)=\sum_{k=0}^{N_{c}-1}\left(\begin{array}{c}
N-1 \\
k
\end{array}\right)\left(s^{*}\right)^{k}\left(1-s^{*}\right)^{N-1-k} .
$$

Note that $p\left(s^{*} ; N_{c}, N\right)$ is a polynomial of degree $N-1$ in $s^{*}$. In particular, $p\left(s^{*} ; N_{c}, N\right)$ is the cumulative distribution function evaluated at $N_{c}-1$ of a binomial distribution with $N-1$ degrees of freedom and probability $s^{*}$. For a mixed strategy Nash equilibrium, $s^{*}$ is a best response for player $i$ only if, given that every other player uses strategy $s^{*}$, player $i$ is indifferent between participating and not participating. That is, at $s^{*}$ we must have

$$
\pi_{i}\left(1, s^{*} ; N_{c}, N\right)=\pi_{i}\left(0, s^{*} ; N_{c}, N\right)
$$

which gives

$$
\alpha+\left(\beta p\left(s^{*} ; N_{c}, N\right)-\gamma\right)=\alpha .
$$

Hence the equilibrium value of $s^{*}$ is implicitly given as the solution of (4), which can be rewritten as:

$$
p\left(s^{*} ; N_{c}, N\right)=\frac{\gamma}{\beta}
$$

The following proposition summarizes the properties of the symmetric MSNE, establishes uniqueness, and gives the relationship between $s^{*}$ and the threshold ratio $b \equiv \frac{N_{c}}{N}$.

Proposition 1 For any $N, N_{c}<N, \alpha, \gamma, \beta>\gamma$, there exists a unique symmetric MSNE $s^{*}$ of the $N$-player participation game with payoff function (1). The value of $s^{*}$ solves (5) and does not depend upon $\alpha$. Moreover, $s^{*} \rightarrow b$ as $N \rightarrow \infty$ for all $\gamma$ and $\beta$, and $s^{*}=1 / 2$ for all $N$ when $b=1 / 2$ and $\gamma / \beta=1 / 2$.

The exact value of $s^{*}$ depends on the threshold value $b$ and on the ratio $\gamma / \beta$. Generically it is unequal to $b$, but it approaches $b$ as the number of players becomes large. This is illustrated in the left panel of Fig. 1 which shows $s^{*}$ as a function of $N$ for $b=\frac{1}{2}$ and different values of $\gamma / \beta$. Furthermore $s^{*}=\frac{1}{2}$ for all $N$ when $b=\frac{1}{2}$ and $\beta=2 \gamma$, that is when the payoff $\alpha$ is exactly in between the payoffs of a successful and a non-successful participation.

Note that, when the game is played repeatedly and agents play the symmetric MSNE, the total number of participating players at time $t, N_{t}$, has mean $N s^{*}$ and variance $N s^{*}\left(1-s^{*}\right)$. The participation rate sequence $\left\{x_{t}\right\}$, with $x_{t}=\frac{N_{t}}{N}$, would therefore be randomly distributed around $s^{*}$ with variance $\frac{s^{*}\left(1-s^{*}\right)}{N}$ and with zero autocorrelations at all lags. Observe that as $N$ becomes large, the distribution of $x$ will converge in probability to a point mass at $s^{*}$ as also illustrated in the right panel of Fig. 1. 

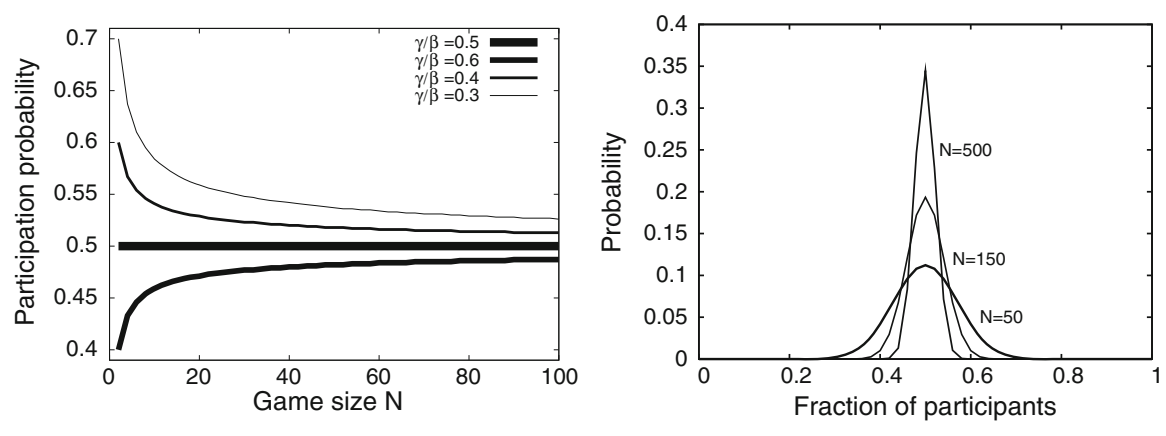

Fig. 1 Symmetric mixed strategy Nash equilibrium. Left panel: every curve shows the MSNE $s^{*}$, for $N$ up to 100 and $b=\frac{N_{c}}{N}=\frac{1}{2}$. Each curve corresponds to a different value of $\gamma / \beta$. Right panel: The distribution of participation rates for three values of $N$ and equal bins of length 0.02 . Other parameters are $b=\frac{N_{c}}{N}=\frac{1}{2}$ and $\frac{\gamma}{\beta}=\frac{1}{2}$

Summarizing, the PSNE has the characteristic that it extracts all rents from the game, but distributes them asymmetrically over the players, whereas the symmetric MSNE gives the same expected payoff to each of the players, but may lead to allocative inefficiencies. $^{4,5}$

\subsection{Experimental Results on Participation Games}

The experimental literature on participation games with negative feedback has dealt with different realizations of this type of strategic interaction. Market entry games, where payoffs for entering a market are linearly decreasing in the number of entrants, have been extensively analyzed in, among others, Sundali et al. (1995); Erev and Rapoport (1998); Rapoport et al. (1998); Zwick and Rapoport (2002) and Duffy and Hopkins (2005). Iida et al. (1992) and Selten et al. (2007) report on laboratory experiments where subjects have to choose between two roads. Meyer et al. (1992) consider suppliers who have to choose between two locations to sell their product and Bottazzi and Devetag $(2003,2007)$ present experimental results on the minority game. ${ }^{6}$ Despite having considered different specifications of participation games with negative feedback, these experimental contributions have put forward the same findings. Aggregate behavior is roughly consistent with the symmetric mixed strategy Nash equilibrium of the underlying one-shot game, with participation rates fluctuating around the optimal capacity of the project. At the individual level, however, subjects typically do not

\footnotetext{
4 The symmetric MSNE is also the unique evolutionary stable strategy (see Dindo 2007).

5 Asymmetric MSNE, with players randomizing with different probabilities, also exist, for example, with $M<N_{c}$ agents always participating and the other $N-M$ agents randomizing with equal probability. In fact, in this case the players randomizing are playing the symmetric MSNE of the participation game with payoff structure (1) but with size $N-M$ and capacity $N_{c}-M$.

6 This game, introduced by physicists (see e.g., Challet and Zhang 1997, 1998 or http://www.unifr.ch/ econophysics), has an odd number of players and positive payoffs only for the players making the minority choice.
} 
Table 1 Experimental results

\begin{tabular}{lllllllll}
\hline & Grp. 1 & Grp. 2 & Grp. 3 & Grp. 4 & Grp. 5 & Grp. 6 & Mean & MSNE \\
\hline Mean $(x)$ & 0.574 & 0.594 & 0.560 & 0.563 & 0.571 & 0.554 & 0.570 & 0.579 \\
Std (x) & 0.157 & 0.127 & 0.182 & 0.174 & 0.194 & 0.184 & 0.169 & 0.187 \\
Grp. switches & 25 & 14 & 25 & 25 & 26 & 22 & 22.8 & 22.9 \\
Ind. switches & 15.7 & 8.3 & 15.6 & 13 & 17 & 14 & 14 & 23.9 \\
\% Naivety & 0.736 & 0.741 & 0.716 & 0.824 & 0.689 & 0.714 & 0.733 & 0.244 \\
\hline
\end{tabular}

The first and second row give, respectively, the average participation rate and its standard deviation for each group. The third row gives the number of times, per group, that participation changed from four or less to five or more subjects or vice versa. The fourth row gives, per group, the number of individual switches between participating and not participating, averaged over subjects. The last row gives the percentage of individual switches that follow directly after a negative payoff experience. The last column gives the corresponding quantities for the symmetric MSNE

adhere to the mixed strategy Nash equilibrium. Bottazzi and Devetag (2007) and Selten et al. (2007), for example, reject the null hypothesis that subjects subsequent choices are independent. In general, individual strategies are heterogeneous, with some subjects always participating, others never participating and many subjects conditioning their behavior on the outcome in previous rounds.

The following experiment on a negative feedback participation game, which we take from Chapter 4 of Heemeijer (2009), nicely illustrates these findings. The experiment involved 6 groups of 7 players, which had to make a participation decision for 50 subsequent periods. The participation game they played could be characterized as $\alpha=100, \beta=50$ and $\gamma=25$, with $N=7$ and capacity set to $N_{c}=4$. Moreover, a stochastic term $\varepsilon$ from a symmetric triangular distribution on $[-25,25]$ was added to the payoff for participating in every period. Group composition remained the same over the course of the experiment. At a PSNE exactly four players participate with certainty and the other three do not. The unique symmetric MSNE is given as $s^{*} \approx 0.5786$, which is slightly larger than the threshold fraction $b=\frac{4}{7} \approx 0.5714$.

Results from this experiment, which was conducted in 2006 at the experimental economics laboratory of the University of Amsterdam, provide an example of the experimental evidence discussed above. The upper left panel of Fig. 2 shows, for the first experimental group, the dynamics of the number of participating subjects over 50 periods. Behavior in the other groups was similar. Aggregate participation decisions are unstable and keep on fluctuating until the end of the experiment. Fluctuations are quite irregular and the upper right panel shows that no significant linear autocorrelation structure can be detected in the participation rate of group 1. Subjects did not coordinate on one of the PSNE and, at the aggregate level, the symmetric MSNE seems to provide a better description of the data. Note that this is achieved even if agents cannot compute the value of $s^{*}$ as, due to the stochastic term $\varepsilon$, they do not know the exact value of the payoffs. The first row of Table 1 shows that average participation rates in all groups are quite close to $s^{*} \approx 0.5786$.

At the individual level, however, the symmetric MSNE is not supported by the data. The lower left panel of Fig. 2, for example, shows a histogram of the number of times the 42 subjects participated. Apparently, some subjects participate almost always, whereas others participate almost never. Further evidence is given by the third and 

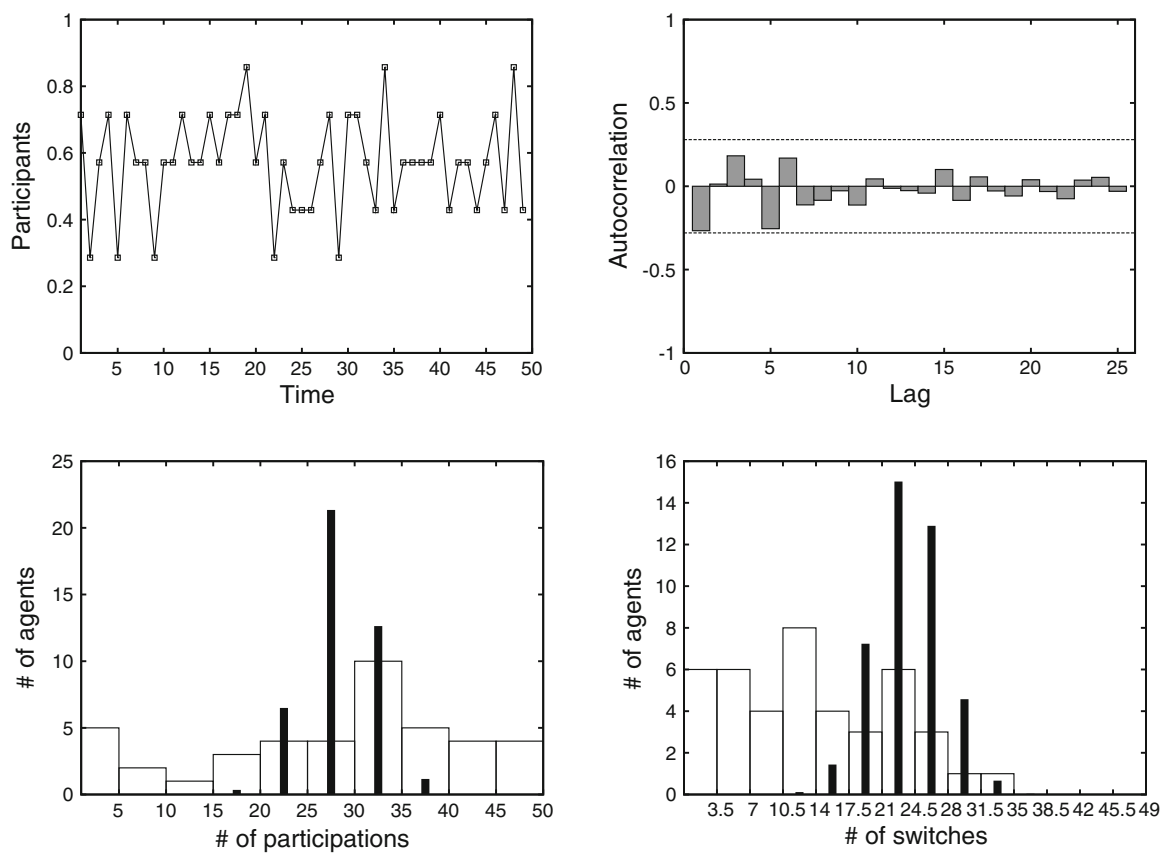

Fig. 2 Upper left panel: time series of number of participating players in experimental group 1. Upper right panel: autocorrelation plot for group 1. Lower left panel: histogram of the number of participations for all 42 participants. Lower right panel: histogram of the number of switches. In both lower panels the vertical thick lines represent the histogram of participations and switches, respectively, implied by the symmetric MSNE

fourth row of Table 1, which show that, although the number of switches in aggregate participation are roughly consistent with the symmetric MSNE, individual subjects change their participation decision much less frequently. The histogram of the number of individual switches, depicted in the lower right panel of Fig. 2 also provides compelling evidence that many subjects are quite reluctant to change. In both histograms of Fig. 2 the differences between the empirical distribution and the distribution where players use $s^{*}$ are striking. Instead of playing according to the symmetric MNSE, subjects seem to condition their decision on past payoffs. The last row of Table 1 shows that about $73 \%$ of individual switches were preceded directly by a negative payoff signal (i.e., start (stop) to participate when participating gave a higher (lower) payoff than not participating in the previous period).

\subsection{Computational Results on Participation Games}

A number of computational models have been advanced to explain the experimental results. For example, Erev and Rapoport (1998) and Duffy and Hopkins (2005) apply a reinforcement learning model to market entry games and find convergence to the mixed strategy Nash equilibrium. Selten et al. (2007) are able to replicate their experimental findings for route choice games by assuming that agents can choose among more complex rules, such as (not) participating if (many) few players participated in the previous round. 
An early influential computational investigation on participation games with negative feedback is advanced by Arthur (1994) who considers $N$ players that independently and repeatedly decide whether to go to a bar or not. The payoff function in this so-called El Farol bar problem is given by (1). Arthur (1994) uses computer simulations to analyze the interaction of 100 agents, each choosing from their own individual set of predictors for the participation rate. Agents make their participation decision on the basis of the selected predictor and past forecasting accuracy of the predictors determines which of them is chosen by the player. Arthur's simulations suggest that: (i) fluctuations persist in the long run, although average participation seems to converge to the capacity $\left(N_{c} / N\right)$ of the bar and (ii) regularities in participation rates are 'arbitraged' away by rules that predict cycles. Zambrano (2004) shows that the average participation rate coincides with the mixed strategy Nash equilibrium of the prediction game that underlies the El Farol bar problem.

A drawback of these computational models is that they are quite complicated and are therefore typically only analyzed by numerical simulations, so that the results may not be robust. This is illustrated by Franke (2003) who applies a reinforcement learning model to the El Farol bar problem. Franke finds that the long run distribution of the probability to participate is either centered around the symmetric mixed strategy Nash equilibrium or is binomial with peaks at very low and very high probabilities to participate. He concludes that the long run outcomes are sensitive to the model's parameter specification. A similar conclusion is drawn by Bell (2001), who also considers the $E l$ Farol bar problem played by agents using a reinforcement learning rule. She shows that different versions of the reinforcement learning rules may lead to dramatically different results. Moreover, in an environment with multiple agents she finds that, although the individual learning rules show erratic behavior, aggregate behavior is quite stable.

\section{A Class of Tractable Evolutionary Models}

In this section we introduce a general model aimed at reproducing the general results coming from the experimental and computational literature surveyed above and that allows both for some analytical tractability and some generality regarding its assumptions. It has two main building blocks. Firstly, we assume that agents use behavioral rules from a general class of rules. These rules are deterministic and condition the decision to participate or not on the outcome of the game in the previous rounds. Secondly, we introduce a general class of updating mechanisms, with the well-known replicator and logit dynamics as special cases, that describes how the fraction of agents using each rule increases with the payoff generated by that rule and with the fraction of agents that is currently using it. These two features are discussed in the next two subsections.

\subsection{A Class of Behavioral Rules}

Each rule prescribes, conditional on the information about the aggregate outcome of past rounds, whether to participate or not. Let $x_{t}$ be the participation rate, i.e., the fraction of players that participates in period $t$. Behavioral rule $K$ has the following form 


$$
p_{k, t}=f_{k}\left(\mathcal{I}_{t-1}\right)
$$

where $f(\cdot)$ is a generic function which maps the information set contains a vector of past participation rates:

$$
\mathcal{I}_{t-1}=\left\{x_{t-1}, x_{t-2}, \ldots, x_{t-L}\right\}
$$

in the decision variable $p_{k, t} \in\{0,1\} .^{7}$ Examples of behavioral rules are:

$$
p_{1, t}=1, p_{2, t}=0 \text { and } p_{3, t}=\left\{\begin{array}{l}
1 \text { if } x_{t-1}<b \\
0 \text { if } x_{t-1} \geq b
\end{array} .\right.
$$

Rule 1, which we denote the optimistic rule, prescribes to always participate, whereas rule 2 , which we denote the pessimistic rule, specifies to never participate. According to rule 3 the player should participate if and only if the participation rate in the previous period turned out to be lower than the threshold value $b$. In fact, all rules above are special cases of a best-response, henceforth BR, rule. Generally, a BR rule can be written as

$$
p_{k, t}=B R\left(g_{k}\left(x_{t-1}, x_{t}, \ldots, x_{0}\right)\right)
$$

where $g_{k}(\cdot)$ is the predictor for the time $t$ participation rate employed by rule $k$ and $B R(\cdot)$ corresponds to the best-responses to that prediction. In other words $B R\left(g_{k}\left(x_{t-1}, \ldots\right)\right)=1$ if and only if $g_{k}(\cdot)<b$, otherwise $B R\left(g_{k}\left(x_{t-1}, \ldots\right)\right)=0$. We will use mainly BR rules in what follows.

\subsection{A Class of Evolutionary Dynamics}

We assume that each rule is used by a fraction of the population of players, that in every period players are randomly matched in groups of $N$ to play the participation game and that, through imitation, a successful rule is adopted by a larger fraction of players the following period. These assumptions can be used to derive a deterministic dynamics from the stochastic system. ${ }^{8}$

Let $x_{k, t}$ denote the fraction of the population using rule $k$ in period $t$. The vector $\mathbf{x}_{t} \in \triangle^{K-1}=\left\{\mathbf{x}_{t} \in \mathbb{R}_{+}^{K}: \sum_{k=1}^{K} x_{k t}=1\right\}$ gives the distribution of players over $K$ rules. At time $t$ the average participation rate across the population of players, $x_{t}$, is

\footnotetext{
7 Note that it would be straightforward to extend the setup to 'randomizing' behavioral rules, by allowing $p_{k, t}$ to be a number between 0 and 1 . However, we are not including this type of rule in our analysis because we want to investigate to what extent deterministic rules are able to reproduce the aggregate random behavior observed in the experimental and computational literature.

8 This approach is often used in evolutionary game theory (see e.g., Benaïm and Weibull 2003 and Sandholm 2003, who show that the deterministic 'mean dynamics' describe the stochastic evolution very well for large populations). Note that it justifies the assumption that each fraction can take on any value in $[0,1]$. Note also that the assumption of a very large number of players can be replaced by the assumption of a very large number of repetitions of the game before the assessment of rules is made.
} 
given by $x_{t}=\mathbf{x}_{t} \cdot \mathbf{p}_{t}=\sum_{k=1}^{K} x_{k, t} p_{k, t}$, that is, it is completely characterized by $\mathbf{x}_{t}$ and $\mathbf{p}_{t}=\left(p_{1, t}, \ldots, p_{K, t}\right)$.

The payoff of rule $k$ is computed as the average of the payoffs which agents using that rule get when they are sorted to play the game. When the number of players in the population goes to infinity, the law of large numbers implies that this average can be computed by assuming that the probability that a player using rule $k$ at time $t$ is given by $x_{k, t}$.

As a result the payoff for rule $k$ at time $t, \pi_{k, t}$, is given by $1-p_{k, t}$ times $\alpha$ plus $p_{k, t}$ times the payoff from participating, which is $\alpha+\beta p\left(x_{t} ; N_{c}, N\right)-\gamma$, where $p\left(x_{t} ; N_{c}, N\right)$ is, as in Eq. 3, the probability that the number of other agents participating is less than $N_{c}$ given the participation rate $x_{t}$. We therefore get

$$
\pi_{k, t}\left(x ; N_{c}, N\right)=\alpha+\left(\beta p\left(x_{t} ; N_{c}, N\right)-\gamma\right) p_{k, t} .
$$

The evolution of the distribution of rules, characterized by the vector $\mathbf{x}_{t}$, is determined by the vector of their payoffs $\pi_{t}$. We model the evolutionary competition between the rules by using a discrete version (see also Rota Bulò and Bomze 2011) of the notion of a revision protocol, that was proposed by Lahkar and Sandholm (2008) and Hofbauer and Sandholm (2009). This gives the map

$$
\mathbf{x}_{t+1}=\mathbf{H}\left(\mathbf{x}_{t}, \pi_{t}\right),
$$

where, for each component $k$, we have

$$
H_{k}\left(\mathbf{x}_{t}, \pi_{t}\right)=x_{k, t}+\sum_{j \neq k} x_{j, t} \rho_{j k, t}-x_{k, t} \sum_{j \neq k} \rho_{k j, t},
$$

and $\rho_{i j, t}$ is the share of strategy $i$ players that switch to strategy $j$ at time $t$. The change in the fraction of agents using rule $k$ is then given by the difference between the inflow into rule $k$, i.e., the total sum of players switching from other rules to rule $k$, and the outflow from rule $k$, i.e., the players switching from rule $k$ to other rules.

We assume that each $\rho_{j k, t}$ can be represented by a non-negative continuous function $\rho_{j k}\left(\mathbf{x}_{t}, \pi_{t}\right)$. Below we impose a number of properties on $\rho_{j k}(\cdot, \cdot)$ to ensure that the switching mechanism is well-behaved.

First, it is easy to check that $0 \leq H_{k}(\mathbf{x}, \pi) \leq 1$, for all $\mathbf{x}$ and $\pi$, provided that we have

$$
\sum_{k \neq j} \rho_{j k}(\mathbf{x}, \pi)<1, \text { for all } j \text { and } k
$$

A second important property is that, for all $j$ and $k$, we have

$$
\rho_{j k}(\mathbf{x}, \pi)\left\{\begin{array}{l}
>0 \text { if } \pi_{k}>\pi_{j} \\
=0 \text { if } \pi_{k} \leq \pi_{j}
\end{array},\right.
$$


that is, a player switches from strategy $j$ to $k$ when the latter results in strictly higher payoffs. Note that this also implies that there is no switching between strategies if they perform equally well.

The third property requires that, for every three strategies $i, j$ and $k$ with $\pi_{j}=$ $\pi_{k}>\pi_{i}$ and defining by $\mathbf{x}_{j+k}$ the vector having $x_{j}+x_{k}$ as its $j$ 'th component and 0 as its $k$ 'th component, it holds that

$$
\rho_{i j}(\mathbf{x}, \pi)+\rho_{i k}(\mathbf{x}, \pi)=\rho_{i j}\left(\mathbf{x}_{j+k}, \pi\right) .
$$

This assumption imposes the intuitive constraint that separating a fraction of the population using a certain rule into separate fractions using essentially the same rule will not change the evolutionary dynamics.

Finally, we impose that for all $j$ and $k$ we have

$$
\left.\rho_{j k}(\mathbf{x}, \pi)\right|_{x_{k}=0}=0,
$$

in fact, it is not possible to imitate a strategy that is not used in the population.

We are now ready to characterize the set of steady states of the evolutionary dynamics. A steady-state of the switching process (9) is a vector $\left(\mathbf{x}^{*}, \mathbf{p}^{*}\right)$ such that, given the rules (6) and the corresponding payoffs $\pi^{*}$ computed through (8), it holds that $\mathbf{x}^{*}=\mathbf{H}\left(\mathbf{x}^{*}, \pi^{*}\right){ }^{9}$ The steady-state participation rate is then given by $x^{*}=\mathbf{x}^{*} \cdot \mathbf{p}^{*}$ where $p_{k}^{*}=f_{k}\left(x^{*}, \ldots, x^{*}\right)$.

Given properties (11-14) we can prove that, independently of the exact specification of the model, the evolutionary dynamics of the behavioral rules introduced in Sect. 3.1 can only exhibit two types of steady states. On the one hand, boundary steady states may exist that correspond to vectors $\mathbf{x}^{*}$ such that $x^{*}=\sum_{k} x_{k}^{*} p_{k}^{*} \in\{0,1\}$. That is, in such a steady state only the optimistic rule, $p=1$, or only the pessimistic rule, $p=0$, is effectively used in the population. An interior steady state, on the other hand, is characterized by a vector $\mathbf{x}^{*}$ such that all rules earn the same, or $\beta p\left(x^{*} ; N_{c}, N\right)=\gamma$. Since this latter condition is equivalent to (5), it follows that all vectors $\mathbf{x}^{*}$ such that $\sum_{k} x_{k}^{*} p_{k}^{*}=s^{*}$ are steady states. The next proposition summarizes the result.

Proposition 2 Consider evolutionary model (9) with behavioral rules (6) and payoffs (8), and assume that the switching functions $\rho_{j k}$ satisfy properties (11-14). Steady states of this model are characterized either by $x^{*} \in\{0,1\}$, or by $x^{*}=s^{*}$, where $s^{*}$ is the symmetric MSNE of the corresponding one-shot game.

Note that boundary and interior steady states correspond to different situations. Boundary equilibria derive from the fact that when all agents are effectively using one rule, they have nobody to imitate, and they cannot switch to a rule that prescribes a different behavior, which is due to property (14). These equilibria therefore typically do not correspond to a Nash equilibrium of the one-shot game. Interior equilibria are

\footnotetext{
9 Notice that for $K$ rules the evolutionary model, as given by Eqs. 6, 8, and 9, can be written as a system of $2 K+L$ difference equations: $K$ for the fractions, $K$ for the rules, and $L$ for the past participations rates, where $L$ is the maximum number of past participation rates used by each rule. Formally, therefore the steady-state is a vector in $\Delta^{K-1} \times\{0,1\}^{K} \times \mathbb{R}_{+}^{L}$.
} 
combinations of fractions and rules where the overall payoff for participating is the same as for not participating, so no more switching occurs in light of property (12). These steady states correspond to the symmetric mixed strategy Nash equilibrium that was discussed in Sect. 2.

The next issue we would like to address are conditions for local stability of the two different types of steady states. The potential for a rigorous local stability analysis is however severely hindered by the fact that many behavioral rules of the type (6) are not differentiable or not even continuous. In fact, the only case which allows for a rigorous local stability analysis is when only optimists and pessimists are present. For more general ecologies of rules, one will have to rely on numerical simulations. In Sect. 4 therefore we will characterize local stability of steady states when all agents are either optimists or pessimists. In Sect. 5, guided by the intuition developed for that benchmark scenario we will investigate the dynamic properties of more complicated versions of the model.

\subsection{Examples of Switching Mechanisms}

When relying on numerical simulations we will need to specify not only the set of rules competing, but also the functional form of the switching function $\rho_{j k}(\cdot, \cdot)$. In this subsection we introduce two types of switching mechanisms satisfying properties (11-14).

The Replicator Dynamics is probably the most widely used updating mechanism in evolutionary economic dynamics. It was originally introduced as a biological reproduction model (see e.g., Taylor and Jonker 1978), where each period the number of agents (i.e., offspring) using a rule grows proportionally with the performance (i.e., fitness) of that rule, as measured by its payoff $\pi_{k}$, but it also arises in imitation processes in large populations of interacting agents (see e.g., Chapter 4 of Weibull 1995 and Schlag 1998). In our setting the replicator dynamics is obtained when choosing

$$
\rho_{j k}(\mathbf{x}, \pi)=x_{k} \frac{\max \left\{\pi_{k}-\pi_{j}, 0\right\}}{\sum_{i=1}^{K} x_{i} \pi_{i}} \forall j, k .
$$

Inserting (15) into (9) and writing $\Delta x_{k, t+1}=x_{k, t+1}-x_{k, t}$ we obtain for each component $K$

$$
\begin{aligned}
\Delta x_{k, t+1} & =\frac{x_{k, t}\left(\pi_{k, t}-\sum_{j} x_{j, t} \pi_{j, t}\right)}{\sum_{j} x_{j, t} \pi_{j, t}} \\
& =\frac{x_{k, t}\left(p\left(x_{t} ; N_{c}, N\right)-\frac{\gamma}{\beta}\right)\left(p_{k, t}-x_{t}\right)}{\frac{\alpha}{\beta}+\left(p\left(x_{t} ; N_{c}, N\right)-\frac{\gamma}{\beta}\right) x_{t}} .
\end{aligned}
$$

which is the standard replicator dynamics equation applied to our framework.

Another well-known updating mechanism is the so-called best response dynamics, where each agent uses a strategy that is a best response to the aggregate outcome of 
the game in the previous round. Since the best response dynamics typically gives rise to a discontinuous mapping an alternative is the smoothed or perturbed best response dynamics (see e.g., Fudenberg and Levine 1998 and Hofbauer and Sandholm 2007), where players choose better responses with higher probabilities. A popular specification of this smoothed best response dynamics is given by the logit dynamics (see e.g., Brock and Hommes 1997, 1998) which corresponds to the switching function

$$
\rho_{j k}(\mathbf{x}, \pi)=\rho_{k}(\pi)=\frac{\exp \left[\pi_{k} / \eta\right]}{\sum_{i=1}^{K} \exp \left[\pi_{i} / \eta\right]} \quad \forall_{j}, k .
$$

Here $\eta>0$ is a smoothing parameter, where for a low value of $\eta(17)$ approximates best response and a high value leads to almost pure and uniform randomization. Note that switching from $j$ to $k$ is independent of which payoff strategy $j$ generated and independent of the distribution of the population over the different strategies, $\mathbf{x}$. Inserting (17) in (9) gives $x_{k, t+1}=\rho_{k}\left(\pi_{t}\right)$. Note however that (17) does not satisfy properties (12), (13) and (14). One consequence of this is that the MSNE does not correspond to a steady state. ${ }^{10}$ For this reason we consider a version of the smoothed best response dynamics which does satisfy our properties, and which we will denote by Adjusted Logit Dynamics. The adjusted logit dynamics uses the following switching function

$$
\rho_{j k}(\mathbf{x}, \pi)=x_{k} \frac{\max \left\{\exp \left[\pi_{k}\right]-\exp \left[\pi_{j}\right]\right\}}{\sum_{i=1}^{K} x_{i} \exp \left[\pi_{i}\right]} \forall j, k,
$$

and it is easily verified that (18) indeed does satisfy properties (11-14). Using (18) we obtain for each component $K$

$$
\begin{aligned}
\Delta x_{k, t+1} & =\frac{x_{k, t}\left(\exp \pi_{k, t}-\sum_{j} x_{j, t} \exp \pi_{j, t}\right)}{\sum_{j} x_{j, t} \exp \pi_{j, t}} \\
= & \frac{x_{k, t}\left(\exp \left[\beta\left(p\left(x_{t}\right)-\frac{\gamma}{\beta}\right) p_{k, t}\right]-x_{t} \exp \left[\beta\left(p\left(x_{t}\right)-\frac{\gamma}{\beta}\right)\right]\right)}{\sum_{j} x_{j, t} \exp \left[p_{j, t} \beta\left(p\left(x_{t}\right)-\frac{\gamma}{\beta}\right)\right]},
\end{aligned}
$$

where $p\left(x_{t}\right)$ is short for $p\left(x_{t} ; N_{c}, N\right)$.

The replicator dynamics (16) depends on payoff differences as well as payoff levels and therefore, for given values of $\gamma / \beta,{ }^{11}$ is not invariant to changes in $\alpha / \beta$. In fact, $\alpha / \beta$ is equivalent with the inverse of the speed of adjustment of the replicator dynamics. ${ }^{12}$ On the other hand, the adjusted logit dynamics (19) only depends upon payoff differences and is therefore independent of $\alpha$. An increase in $\beta$, however, increases payoff differences and therefore increases the rate with which fractions change. This parameter is therefore related to the intensity of choice $\left(\eta^{-1}\right.$ in Eq. 17).

\footnotetext{
10 Also the boundary steady states disappear for the smoothed best response dynamics.

11 Recall that, for a given ratio $\gamma / \beta$, the set of Nash equilibria is independent of both $\alpha$ and $\beta$ (see Sect. 2).

12 In particular, when $\alpha / \beta \rightarrow \infty$ trajectories of the discrete dynamical system (16) approach the trajectories of the corresponding continuous time dynamical system (see e.g., Chap. 4 in Weibull 1995).
} 


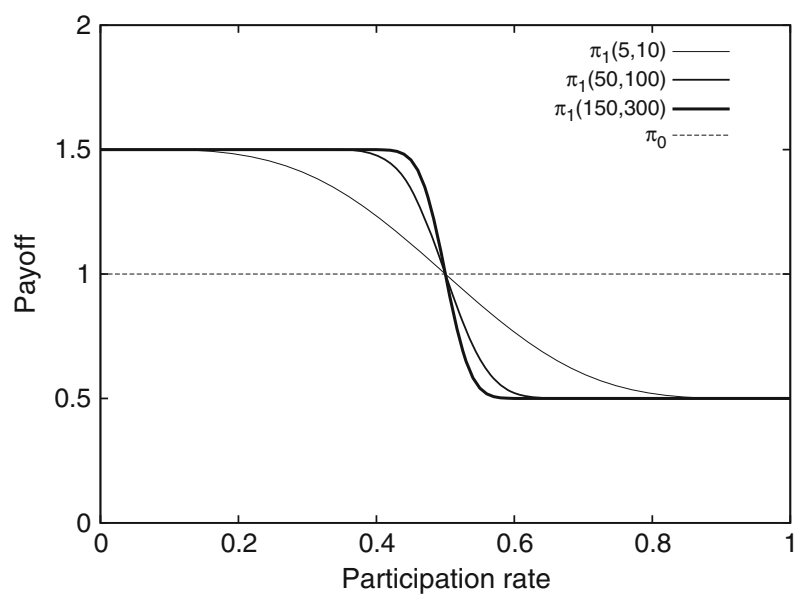

Fig. 3 Payoffs of the pessimistic rule (21) (horizontal line) and optimistic rule (20) for different values of $N$. The other parameters are $\alpha=1, \gamma=\frac{1}{2}, \beta=2 \gamma$ and $b=\frac{1}{2}$

\section{Optimists Versus Pessimists}

Consider the model of evolutionary competition with the following two rules

$$
\begin{aligned}
& p_{1, t}=1 \text { for all } t, \quad \text { and } \\
& p_{0, t}=0 \text { for all } t .
\end{aligned}
$$

The optimistic rule (20) prescribes to always participate and the pessimistic rule (21) prescribes to never participate. The experimental evidence discussed in Sect. 2 suggests that many subjects use one of these rules for a substantial number of periods. Rules (20) and (21) can also be interpreted as best-response rules (7), with (20) the best reply to an optimistic predictor (always predicting below $b$ ), and (21) the bestreply rule to a pessimistic predictor. Given the rules, payoffs follow straightforwardly from (8) as $\pi_{1, t}=\alpha+\beta p\left(x_{t} ; b N, N\right)-\gamma$ for the optimists and $\pi_{0, t}=\alpha$ for the pessimists. Figure 3 plots both as a function of $x$ for $b=\frac{1}{2}$ and different values of $N$. Note that due to the negative feedback expected payoffs for the optimistic rule are decreasing in the number of participants. As $N$ becomes large, the optimistic payoff $\pi_{1, t}$ converges to the step payoff function given in (1). On the other hand, when $N$ is small the expected payoff function is less steep.

Using $x_{t}=x_{0, t} p_{0, t}+x_{1, t} p_{1, t}=x_{1, t}$ and $x_{0, t}=1-x_{1, t}$ our only state variable is $x_{t}$ and the evolutionary model (9-10) can be written as

$$
x_{t+1}=x_{t}+\left(1-x_{t}\right) \rho_{01, t}-x_{t} \rho_{10, t}
$$

where $\rho_{01, t}=\rho_{01}\left(x_{t}, \pi_{0, t}, \pi_{1, t}\right)$ and $\rho_{10, t}=\rho_{10}\left(x_{t}, \pi_{0, t}, \pi_{1, t}\right)$ depend upon $x_{t}$ directly as well as indirectly through the profit function $\pi_{1, t}$. 

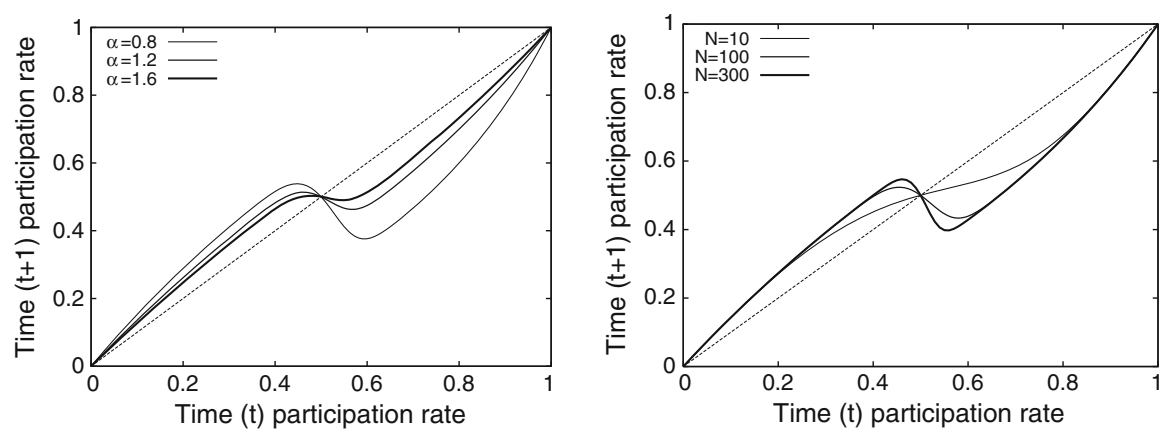

Fig. 4 Graph of replicator dynamics (23) for three different values of $\alpha$ and N. Left panel: map for three different values of $\alpha$ and $N=100$. Right panel: map for three different values of $N$ and $\alpha=1$. In both panels the other parameters are $\gamma=\frac{1}{2}, \beta=2 \gamma$ and $b=\frac{1}{2}$

In case of the replicator dynamics we obtain

$$
x_{t+1}=\frac{x_{t}\left(\frac{\alpha}{\beta}+p\left(x_{t} ; b N, N\right)-\frac{\gamma}{\beta}\right)}{x_{t}\left(p\left(x_{t} ; b N, N\right)-\frac{\gamma}{\beta}\right)+\frac{\alpha}{\beta}},
$$

whereas under the adjusted logit dynamics we have

$$
x_{t+1}=\frac{x_{t} \exp \left\{\beta\left(p\left(x_{t} ; b N, N\right)-\frac{\gamma}{\beta}\right)\right\}}{x_{t} \exp \left\{\beta\left(p\left(x_{t} ; b N, N\right)-\frac{\gamma}{\beta}\right)\right\}+\left(1-x_{t}\right)} .
$$

In both cases the dynamics can be described by a first order nonlinear difference equation parameterized by $N, b=\frac{N_{c}}{N}, \alpha, \beta$ and $\gamma$. In the panels of Figs. 4 and 5 we plot (23) and (24) for different values of the number of players $N$ and the parameters $\alpha$ or $\beta$, respectively.

By Proposition 2, and as illustrated by Figs. 4 and 5, the three steady states are given by $x^{*}=0, x^{*}=1$ and the symmetric mixed symmetric Nash equilibrium $x^{*}=s^{*}$. To characterize local stability of these steady states it is convenient to define

$$
\delta(x ; b, N)=\frac{\partial p(x ; b N, N)}{\partial x} .
$$

Obviously $\delta(x ; b, N)<0$, since an increase in the fraction of agents participating always decreases the probability that less than $b N$ of them indeed participate. Local stability analysis also requires that we make some assumptions on the derivatives of the switching function. In particular we make the intuitive assumption that for $j, k=0,1$ we have

$$
\left.\frac{\partial \rho_{j k}(x, \pi)}{\partial x_{k}}\right|_{x_{k}=0, \pi_{k}>\pi_{j}}>0 .
$$



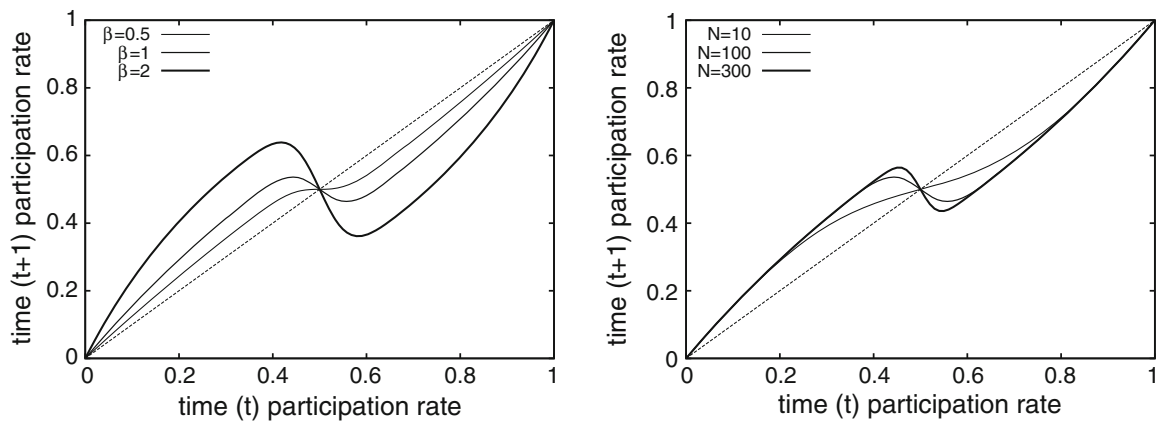

Fig. 5 Graph of adjusted logit dynamics (24) for three different values of $\beta$, keeping $\gamma / \beta$ fixed, and $N$. Left panel: map for three different values of $\beta$, keeping $\gamma / \beta$ fixed and $N=100$. Right panel: map for three different values of $N$ and $\beta=1$. In both panels the other parameters are $\beta=2 \gamma$ and $b=\frac{1}{2}$

That is, if the fraction using strategy $k$ increases from zero, then more agents will move to that strategy. We also impose

$$
\left.\frac{\partial \rho_{j k}(x, \pi)}{\partial_{+} \pi_{k}}\right|_{x_{k}>0, \pi_{k}=\pi_{j}}>0 \text { and }\left.\frac{\partial \rho_{j k}(x, \pi)}{\partial-\pi_{j}}\right|_{x_{k}>0, \pi_{k}=\pi_{j}}<0,
$$

where $\partial_{+}$and $\partial_{-}$refer to right and left derivatives respectively. ${ }^{13}$ These assumptions imply that, when payoffs are equal, an increase in the payoff differential increases the propensity to switch.

The following proposition characterizes stability of the steady states for our general class of evolutionary dynamics when all agents are either optimists or pessimists.

Proposition 3 Consider the participation rate dynamics given by (22) and suppose properties (11-14) and properties (25-26) hold. Then the two boundary steady states $x^{*}=0$ and $x^{*}=1$ are locally unstable. The interior equilibrium $x^{*}=s^{*}$ is locally stable when both the right derivative

$$
J_{+}\left(s^{*}\right)=1+\left.\left(1-s^{*}\right) \beta \delta^{*} \frac{\partial \rho_{01}}{\partial_{+} \pi_{1}}\right|_{x=s^{*}}
$$

and the left derivative

$$
J_{-}\left(s^{*}\right)=1-\left.s^{*} \beta \delta^{*} \frac{\partial \rho_{10}}{\partial_{-} \pi_{1}}\right|_{x=s^{*}}
$$

are larger than -1 , where $\delta^{*} \equiv \delta\left(s^{*} ; b, N\right)$.

13 We need to use right and left derivatives because, due to property (12), the switching function $\rho_{j k}$ has a kink at $\pi_{j}=\pi_{k}$, and left and right derivatives are therefore not equal at that point (in particular, the right derivative with respect to $\pi_{j}$ and the left derivative with respect to $\pi_{k}$ are both equal to zero at $\pi_{j}=\pi_{k}$ ). 
It is now easy to apply the previous proposition for specific choices of switching functions satisfying (25-26), like those corresponding to the replicator or adjusted logit dynamics. Applying Proposition 3 to both dynamics, and upon noticing that for both we have $J_{+}\left(s^{*}\right)=J_{-}\left(s^{*}\right)=J\left(s^{*}\right)$, we obtain the following result

Corollary 1 The interior steady state $s^{*}$ is locally stable for the replicator dynamics (16) when

$$
J\left(s^{*}\right)=1+s^{*}\left(1-s^{*}\right) \frac{\beta \delta^{*}}{\alpha}>-1
$$

and $s^{*}$ is locally stable for the adjusted logit dynamics (19) when

$$
J\left(s^{*}\right)=1+s^{*}\left(1-s^{*}\right) \beta \delta^{*}>-1 .
$$

The stability conditions in Proposition 3 and Corollary 1 depend upon $\beta$, possibly $\alpha$ and, through $\delta^{*}$ and $s^{*}$, implicitly upon $b$ and $N$. Figure 6 shows the dynamics of $x$ and the corresponding Lyapunov exponent for different values of $\alpha$ for the replicator dynamics. For a high speed of adjustment ( $\operatorname{small} \alpha$ ), an overshooting effect causes instability. As the speed of adjustment decreases (i.e., as $\alpha$ increases) adaptation is slower and the equilibrium $s^{*}$ becomes stable. The right panel of Fig. 6 indicates that there exist many values of $\alpha$ for which the dynamics is chaotic, in the sense that it exhibits sensitive dependence upon initial conditions. ${ }^{14}$ Also note that the dynamics is invariant under a rescaling of the payoffs $\alpha, \beta$ and $\gamma$, implying that a unilateral increase from $\alpha$ to $\vartheta \alpha$ is equivalent with a change from $\beta$ and $\gamma$ to $\beta / \vartheta$ and $\gamma / \vartheta$, respectively. Figure 6 therefore also illustrates the effect of a simultaneous decrease in $\beta$ and $\gamma$. The left panel of Fig. 8 shows what happens in the adjusted logit dynamics when $\beta$ increases. Differently from the replicator dynamics case, when the interior steady state $s^{*}$ becomes unstable, the dynamics converges to a period two cycle for all values of $\beta$.

We are also interested in characterizing the stability of $s^{*}$ as a function of $N$, which affects the stability both through $s^{*}$ and $\delta^{*}$. The right panels of Figs. 4 and 5 already suggests that $s^{*}$ becomes unstable for $N$ large enough. The following proposition corroborates that.

Proposition 4 Let the dynamics of participation rates be given by (22). For any given value of $\alpha, \gamma<\beta$, and $b$ the interior steady state $s^{*}$ is unstable when $N \rightarrow \infty$. Moreover, if $\beta=2 \gamma$ and $b=\frac{1}{2}$ there exists a unique $M$ such that the steady state ${ }^{*}$ is locally stable if and only if $N<M$.

Figure 7 shows the dynamics of $x$ and the corresponding Lyapunov exponent for different values of $N$ for the replicator dynamics, and the right panel of Fig. 8 shows

14 The left panel of Fig. 6 also shows that for some parameter values (for example, for $\alpha \approx 0.8$ ) this one-dimensional system has a 3-cycle which implies, by the Li-Yorke theorem (1975) that the dynamics for those parameter values is chaotic. 

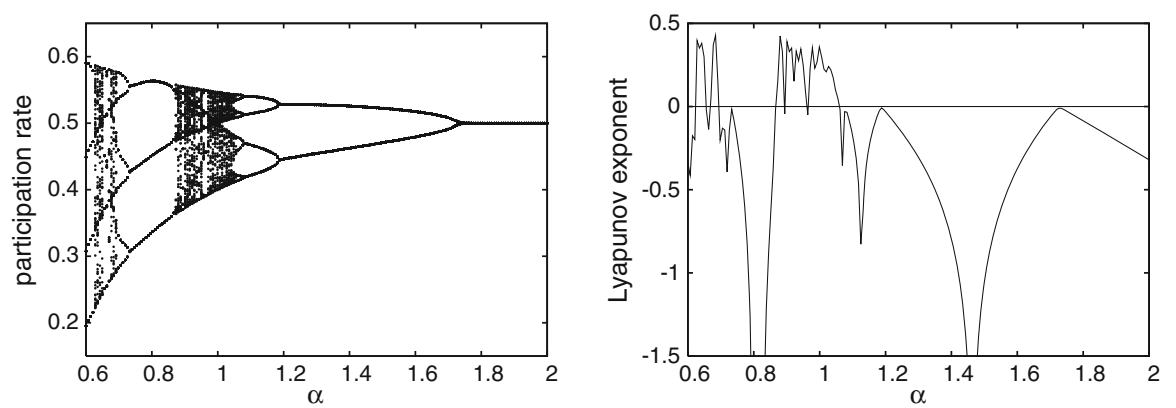

Fig. 6 Replicator dynamics. Left panel: bifurcation diagram with respect to $\alpha$. Right panel: Lyapunov exponents for different values of $\alpha$. Parameters values are $b=\frac{N_{c}}{N}=\frac{1}{2}, N=300, \gamma=\frac{1}{2}$ and $\beta=2 \gamma$. For every value of $N, 100$ iterations are shown after an initialization period of 100
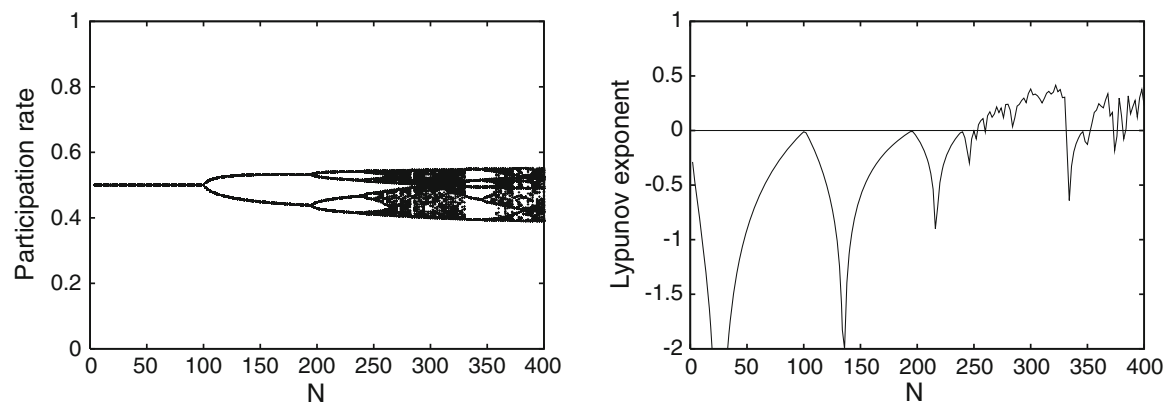

Fig. 7 Replicator dynamics. Left panel: bifurcation diagram with respect to $N$. Right panel: Lyapunov exponents for different values of $N$. Parameters values are $b=\frac{N_{c}}{N}=\frac{1}{2}, \alpha=1, \gamma=\frac{1}{2}$ and $\beta=2 \gamma$. For every value of $N, 100$ iterations are shown after an initialization period of 100
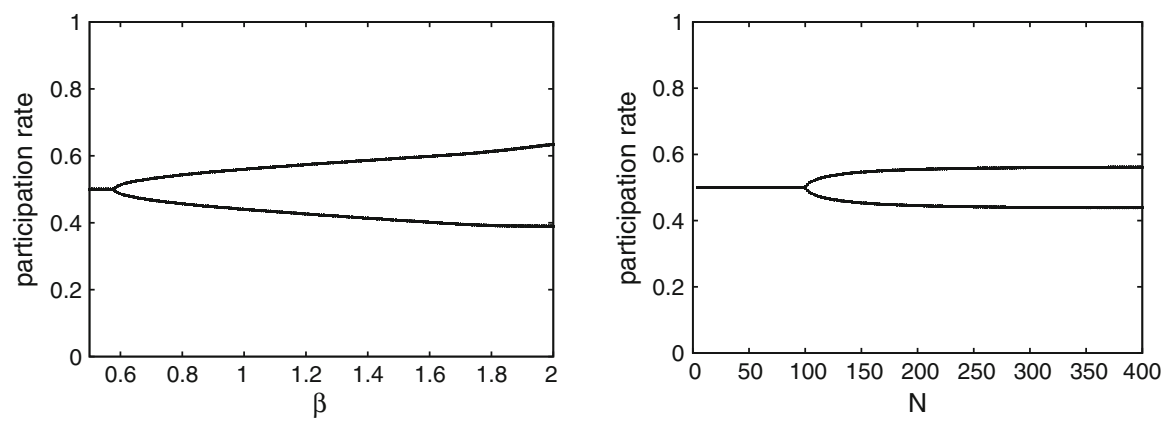

Fig. 8 Adjusted logit dynamics. Left panel: bifurcation diagram with respect to $\beta$, keeping the ratio $\gamma / \beta$ fixed. Right panel: bifurcation diagram with respect to $N$. Parameters values are $b=\frac{N_{c}}{N}=\frac{1}{2}, N=300$, and $\beta=2 \gamma$. For every value of $N, 100$ iterations are shown after an initialization period of 100

the dynamics of $x$ for different values of $N$ for the adjusted logit dynamics. For small $N$ the dynamics is stable but as $N$ increases an overshooting effect causes instability. Indeed Fig. 9 shows that $J\left(s^{*}\right)$ is decreasing in $N$ and crosses the horizontal line with intercept -1 when $N$ is larger than some threshold value $M$. This holds both for the 

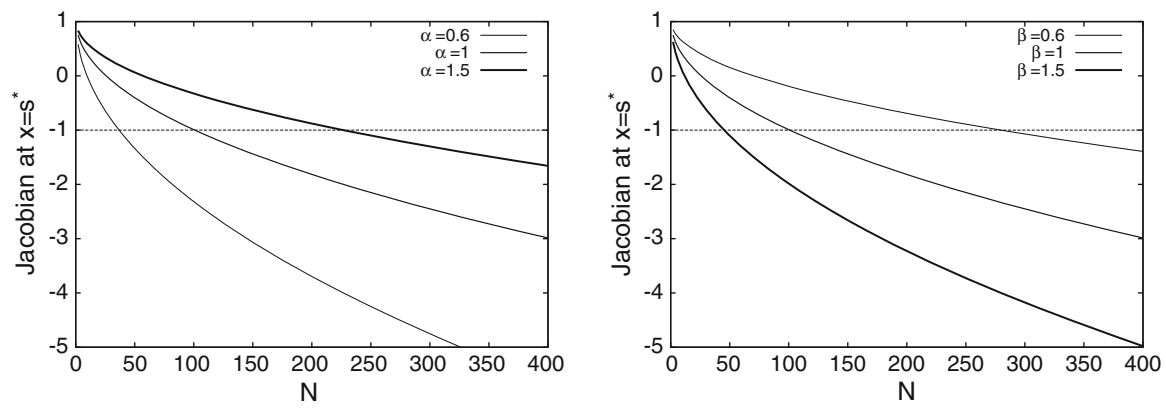

Fig. 9 Stability condition. Slope of (22), evaluated at $x=s^{*}$, as a function of $N$, for two types of switching dynamics. Left panel: replicator dynamics. Right panel: adjusted logit dynamics. When not otherwise specified parameters are $b=0.5, \beta=1$ and $\alpha=1$

replicator dynamics (left panel) and for the adjusted logit dynamics (right panel). The intuition behind Proposition 4 is that as $N$ increases the average population payoff of the optimistic rule gets closer to the step payoff function of the underlying one shot game (see Fig. 4). As a result, for any value of the payoff parameters, $\alpha, \beta>\gamma$, as $N$ increases (22) becomes steeper at the steady state $s^{*}$ and the system loses stability. This dependence of the dynamics upon $N$ is due to the assumption of random matching. In fact, for $N=2$ (with $N_{c}=1$ ) the optimists payoff, $\pi_{1}$, is linear in $x$, since the probability of meeting a player who is using the pessimistic rule is $p(x ; 1,2)=1-x$. However, the probability of having less than half of the players participating when more than half of the other players are optimists, decreases as $N$ increases. That is, as $N$ increases (and for given $b$ ) the function $p(x ; b N, N)$ will look more and more like a step function. We can therefore also interpret the parameter $N$ as a measure of the shape and steepness of the payoff function at the steady state in a framework where all the (large) population of players play the same game. In that case a low value of $N$ would give an optimists payoff function which decreases slowly as the number of participating players increases, similar to the linear one used in the market entry experiments (see, for example, Sundali et al. 1995). A high value of $N$, on the other hand, would represent an expected payoff function close to the step function used in the El Farol bar game, with payoffs at the symmetric MSNE dropping rapidly as an extra player participates.

Figure 10 gives a specific example of the global dynamics of the replicator dynamics when the steady state $s^{*}$ is locally unstable. The time series (left panel) looks aperiodic and indeed the Lyapunov exponent for these values of the parameters is positive so that the dynamics exhibits sensitive dependence on initial conditions (see right panel of Fig. 6 at $\alpha=1$ or right panel of Fig. 7 at $N=300$ ).

Even such a simple one-dimensional system is therefore able to produce complicated times series similar to those obtained by, e.g., the computational model of Arthur (1994). The evidence is gathered in the panels of Figs. 6 and 7. Clearly, as $\alpha$ decreases and/or $N$ increases the dynamics of the participation rate $x$ becomes unstable, after which a period-doubling route to chaos sets in, at least for the replicator dynamics. The right panels show that there exist values of both parameters where the Lyapunov 

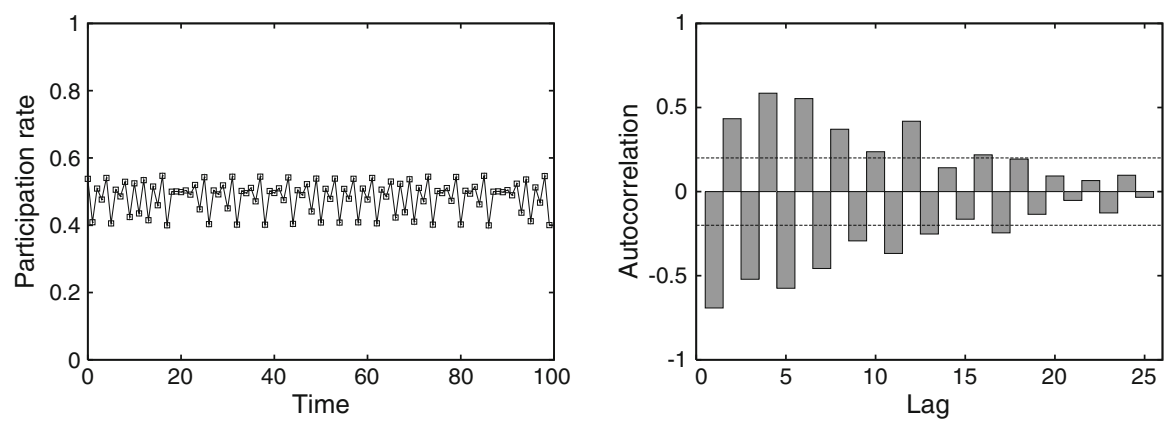

Fig. 10 Replicator dynamics. Left panel: participation rate time series. Right panel: autocorrelation diagram. Parameters values are $N=300, N_{c}=150, \alpha=1, \gamma=\frac{1}{2}$ and $\beta=2 \gamma$

exponents is positive implying sensitive dependence on initial conditions, a possible definition of chaotic behavior.

Finally, note that the influence of $\alpha, \beta$ and $N$ on the dynamics is at odds with what should be expected if agents were repeatedly playing the MSNE of the one-shot game. First, increasing $\alpha$ destabilizes the dynamics in our model, although it does not have an impact on the equilibria of the one-shot game (see footnote 3 in Sect. 2). Second, fluctuations in the participant rate in our numerical simulations are increasing (or at least not decreasing) when the number of players $N$ increases, whereas in Sect. 2 we have shown that at the symmetric MSNE the variance of the participation rate approaches zero as the number of players increases. Experiments with human subjects and different values of $N$ and $\backslash$ or $\alpha$ may shed more light on the real relationship between these parameters and the size of the fluctuations, and thus on the validity of our model.

\section{Exploiting Participation Fluctuations}

In the previous section we have established that for a high speed of adjustment, a high intensity of choice, and/or for large $N$, the evolutionary competition between an optimistic and a pessimistic rule induces fluctuations of the aggregate participation rate $x$ around the steady state $s^{*}$. The right panel in Fig. 10 shows that there may be a significant correlation between the participation rates and its lagged values. One might argue that the competition between the optimistic and pessimistic rule is not stable against the entrance of more sophisticated rules. The aim of this section is to investigate how the inclusion of such new rules affects the participation rate dynamics and whether they may succeed in exploiting these regularities. Similar analyses have been performed in Hommes (1998) and Brock et al. (2006) for the cobweb model. Most of our analyses in this section make use of the replicator dynamics (16) as updating mechanism, but we will also briefly discuss the results when fractions are updated according to the adjusted logit dynamics (19).

Since there is a strong positive autocorrelation at the second lag, indicating 'upand-down' behavior, a best response to predicting $x_{t-2}$ for period $t$ seems to be a 
sensible strategy. This so-called "2-lags best reply" is given by 15

$$
p_{2, t}=B R\left(x_{t-2}\right)=\left\{\begin{array}{l}
1 \text { if } x_{t-2}<b \\
0 \text { if } x_{t-2} \geq b
\end{array} .\right.
$$

Generalizing this to three lags, four lags, or $j$ lags best responders is straightforward. Letting $x_{2, t}$ be the fraction of 2-lags best responders, the participation rate at time $t$ is given as:

$$
x_{t}=x_{1, t}+x_{2, t} B R\left(x_{t-2}\right),
$$

where $x_{1, t}$ and $x_{0, t}=1-x_{1, t}-x_{2, t}$ are the fractions of optimists and pessimists, respectively. As before, the latter have payoffs $\alpha$ and expected payoff for optimists is given by $\pi_{1, t}=\alpha+\beta p\left(x_{t} ; N_{c}, N\right)-\gamma$. Expected payoffs for 2-lags best responders are:

$$
\pi_{2, t}=\left(1-B R\left(x_{t-2}\right)\right) \alpha+B R\left(x_{t-2}\right) \pi_{1, t} .
$$

Now let fractions $x_{0, t+1}, x_{1, t+1}$ and $x_{2, t+1}$ evolve according to the replicator dynamics (16), which is based upon payoffs generated by the different rules, which, in turn, depend upon $x_{t}$ and $x_{t-2}$. This leads to a four-dimensional dynamical system. The interior steady state of this system is characterized by $x=s^{*}$. At $x=s^{*}$ every rule generates the same expected payoff. If $s^{*} \geq b$ the two lags best responders are not participating at the steady state. Therefore, there is a continuum of steady state fractions with $x_{1}^{*}=s^{*}$ and $x_{0}^{*}+x_{2}^{*}=1-s^{*}$.

Because the rule $p_{2, t}$ is discontinuous we rely on numerical simulations to determine local stability of these steady states. As illustrated in the upper panels of Fig. 11, the replicator dynamics with these rules is unstable and leads to persistent fluctuations in participation rates for a high speed of adjustment (low $\alpha$ ) and/or large $N .{ }^{16}$ The lower panels of Fig. 11 show the time average of the fraction of 2-lags best responders along 100 iterations. This average fraction approaches zero when the steady state $s^{*}$ is locally stable and is strictly positive otherwise. With high $\alpha$ and small $N$ the dynamics converges to the steady state $x=s^{*}$ along a "shrinking" 3-cycle. Along such a cycle a 2-lags best responder is more often wrong than right in its prediction and slowly disappears. Note that, even if $x_{2}$ approaches zero, it could happen that the participation rate $x$ settles at $s^{*}$ before $x_{2}$ equals zero. The simulations show that this typically does not happen.

\footnotetext{
15 The reader may wonder why we do not investigate a "1-lag best reply". Since the interaction between the optimistic and the pessimistic rule generates a strong first order negative autocorrelation in participation rates, one lag best responders are very often wrong in their prediction. Simulations confirm that these one lag best responders are quickly driven out.

16 Simulations show that the instability threshold of both $\alpha$ and $N$ are roughly the same as those found in the analysis of optimists vs. pessimists in Sect. 4. Back of the envelope computations for a continuous approximation of (29) show that, provided $B R^{\prime}\left(s^{*}\right)=0$, local stability of $s^{*}$ is still related to the conditions given in Prop. 3.
} 

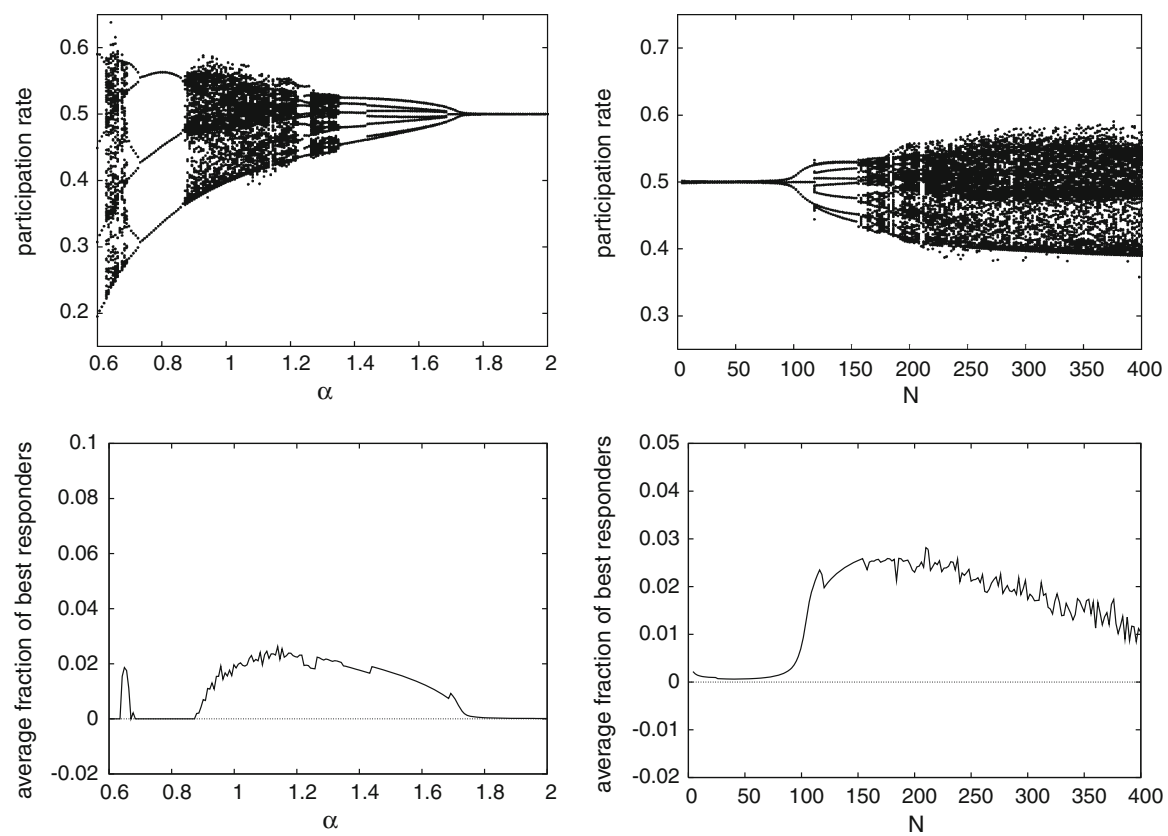

Fig. 11 Replicator Dynamics. Evolutionary competition between optimists, pessimists and two lags best responders. Top left panel: long run participation rate for different speeds of adjustment $\alpha$. Bottom left panel: time average (over 100 periods) of the fraction of 2-lags best responders for different values of $\alpha$. The time average is computed along the iterations shown in the top left panel. Right panels: same as above but with changing number of players $N$ and fixed $\alpha$. In all the cases $b=0.5 \beta=2 \gamma$ and $\gamma=0.5$

A different participation rate dynamics occurs when $\alpha$ is low and $N$ large. In this case the dynamics is typically non-periodic and 2-lags best responders survive. Moreover the stable 2-cycles that existed when only optimists and pessimists are present have now disappeared. In fact, no 2-cycle could exist in this framework with 2-lags best responders. Assuming that a 2-cycle exists implies that the fraction of 2-lags best responders is either always increasing, at the expense of both optimists and pessimists, or already equal to one. In the first case the 2-cycle is not stable, since fractions are changing, in the second case all agents are 2-lags best repliers so that the 2-cycle must be something like $\{0,1,0,1, \ldots\}$. But this is in contradiction with the 2-lags BR rule since when recording such a sequence the rule would predict that the next period few players will participate, thus suggesting to participate. This leads to $0,1,1$, and therefore the 2-cycle is broken up.

The absence of 2-cycles is also reflected in the autocorrelation structure of the chaotic dynamics. The upper left panel of Fig. 13 shows the participation rate for $N=300$. Even though the time series is not periodic, the autocorrelation diagram (upper right panel of Fig. 13) shows that the autocorrelation at the second lag has indeed decreased substantially (and in fact is not significantly different from zero).

Despite the fact that 2-cycle behavior seems to have disappeared from the autocorrelation plot, the autocorrelation at the third lag is strongly positive, suggesting that the time series of participation rates has elements of a noisy 3-cycle. 

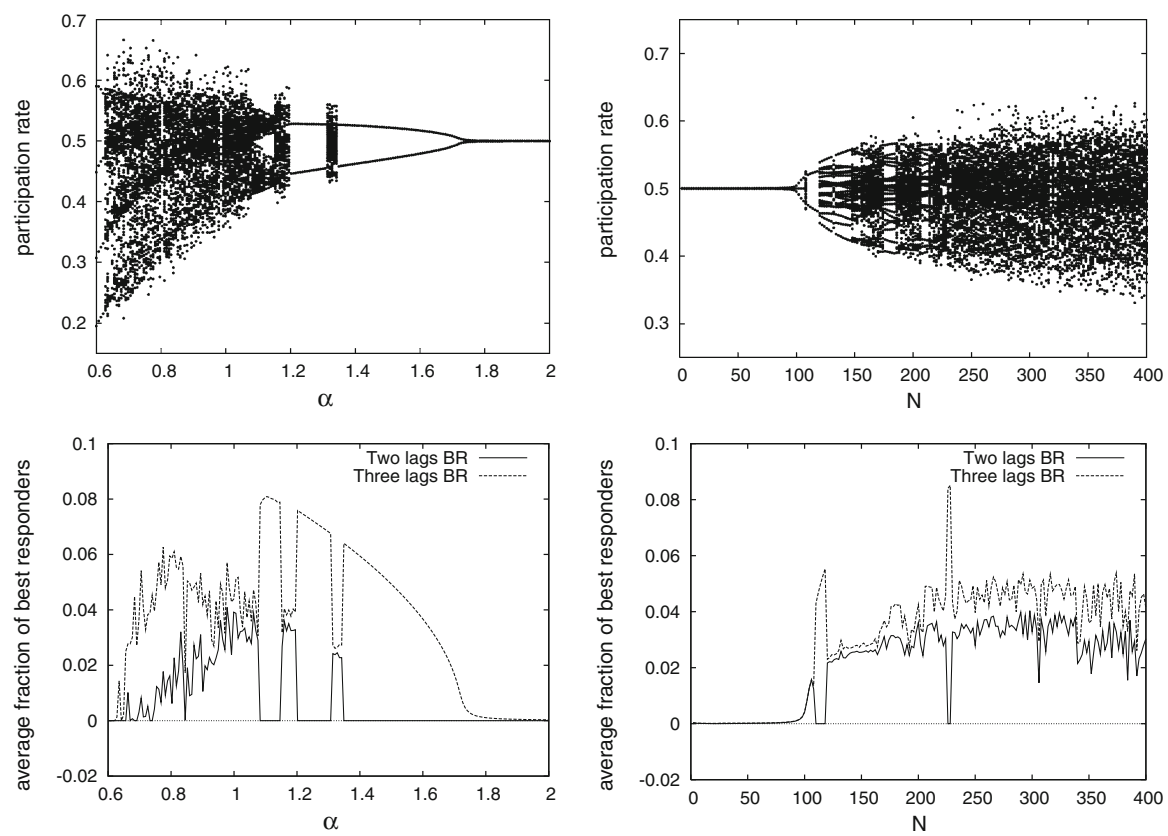

Fig. 12 Replicator Dynamics. Evolutionary competition between optimists, pessimists, two and three lags best responders. Top left panel: long run participation rate for different speeds of adjustment $\alpha$. Bottom left panel: time average (over 100 periods) of the fraction of 2-lags best responders for different values of $\alpha$. The time average is computed along the iterations shown in the top left panel. Right panels: same as on the left but with changing number of players $N$ and fixed $\alpha$. In all the cases $b=0.5$ and $\beta=2 \gamma$

This begs the question as to what would happen if a new rule that tries to exploit this feature is introduced. To that end, we introduce the " 3 -lags best reply" $p_{3, t}=$ $B R\left(x_{t-3}\right)$. The evolutionary competition of the four rules gives a six-dimensional dynamical system. Given that $s^{*} \geq b$ there is a continuum of steady states of the type $x_{1}=s^{*}$ and $x_{0}+x_{2}+x_{3}=1-s^{*}$. The steady state $s^{*}$ is unstable for low $\alpha$ and large $N$ and leads to erratic participation rates, as can be seen in the middle-upper left panel of Fig. 13. The upper and lower panels of Fig. 12 show, respectively, the participation rate dynamics and the average fraction (over 100 iterations) of two and three lags best responders when $\alpha$ and $N$ are changing. When $\alpha$ is large, or $N$ is small, the participation rate converges to $s^{*}$ and the fractions of both types of best responders go to zero. This is because the participation rate converges to $x=s^{*}$ along 4-cycles of decreasing amplitude, along which both types of best responders are more often wrong than right in their predictions. Instead, when $\alpha$ is small or $N$ is large, the dynamics is unstable ${ }^{17}$ and both types of best responders may survive. Also in this case there exist values of the parameters where the dynamics looks aperiodic and cycles of period 2 and 3 have

\footnotetext{
17 Back of the envelope computations show that the stability conditions the dynamics under a set of continuous versions of the best reply rule, are the same as those found when only optimists, pessimists, and 2-lags best repliers are playing. See footnote 16 .
} 

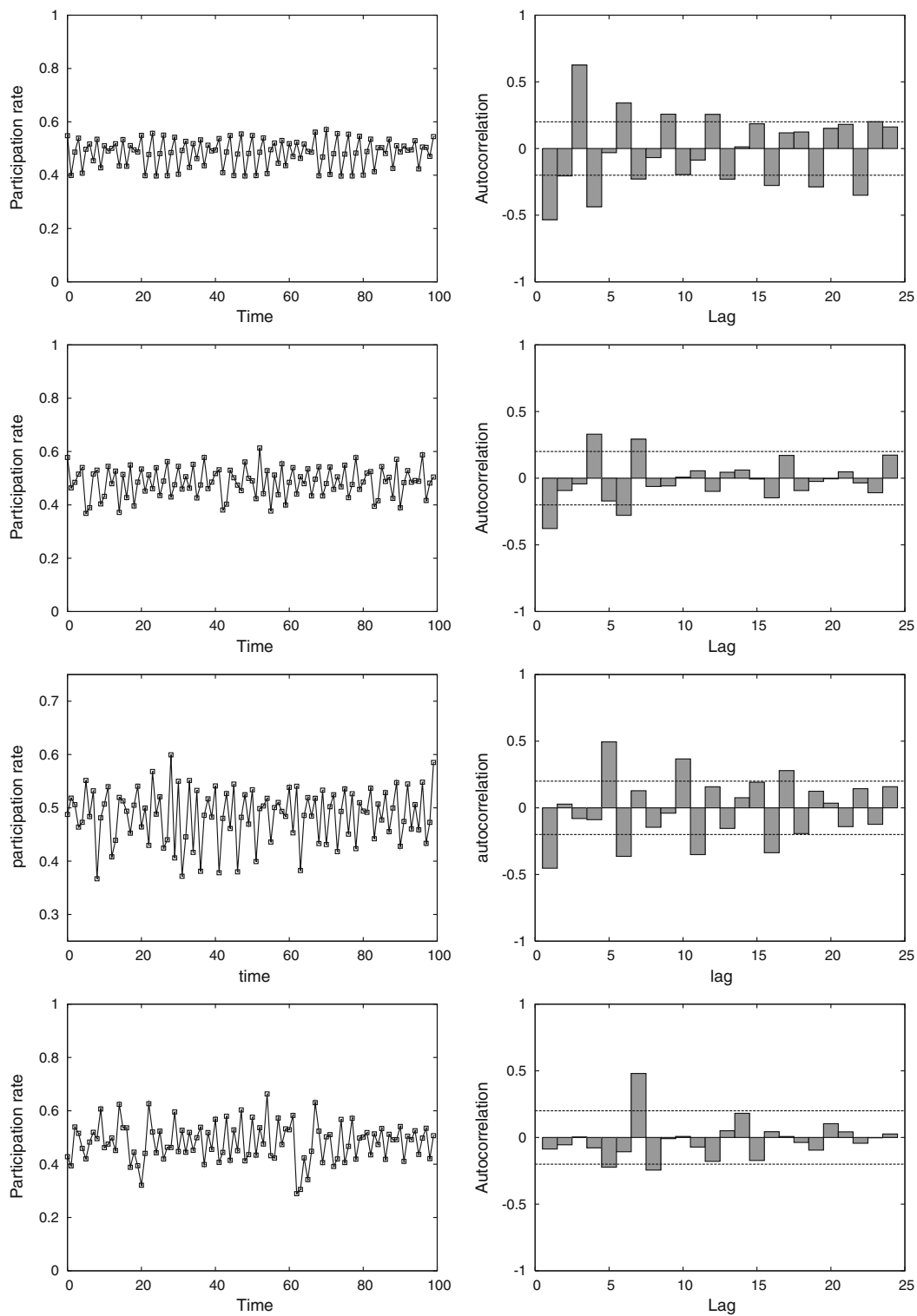

Fig. 13 Replicator dynamics. Upper panels: participation rate and autocorrelation diagram for competition between optimists, pessimists, and 2-lag best responders. Middle-upper panels: participation rate and autocorrelation diagram for competition between optimists, pessimists, two, and three lag best responders. Middle-lower panels: participation rate and autocorrelation diagram for competition between optimists, pessimists, two, three, and four lag best responders. Lower panels: participation rate and autocorrelation diagram for competition between optimists, pessimists, and two to six lag best responders. The dotted lines show the 5\% significance levels of the autocorrelations. Autocorrelations and significance levels are based upon a sample of 100 observations. Parameters values are $N=300, b=\frac{1}{2}, \alpha=1, \beta=2 \gamma$ and $\gamma=\frac{1}{2}$ 
disappeared. ${ }^{18}$ It is interesting to notice that typically these more sophisticated best response rules are driven out when the dynamics is stable, with only the optimistic and pessimistic surviving, and thrive when the dynamics is unstable.

The autocorrelation diagram depicted in the middle-upper right panel of Fig. 13 suggests that $x_{t-4}$ would be a good predictor of $x_{t}$. Again therefore, the evolutionary competition leads to a regularity in participation rates that cannot be exploited by the rules that are present in the population. Adding new rules does not stabilize the dynamics, it merely drives out one regularity at the expense of introducing higherorder regularities. This is illustrated by the lower panels of Fig. 13 where the results are shown of the replicator dynamics with the optimistic rule, the pessimistic rule, and two, three, four, five and six lags best responders, for $N=300$. The participation rate still exhibits perpetual fluctuations, in this case with positive autocorrelation at the 7th lag. Autocorrelation at lower lags is exploited efficiently by the existing behavioral rules and is not significantly different from zero.

The numerical simulations presented thus far in this section all made use of the replicator dynamics as updating mechanism. Recall that the dynamic behavior for the adjusted logit dynamics was very regular when only optimist and pessimist behavioral rules were available: the dynamics converged either to the interior steady state $s^{*}$, or to a period two cycle (see Fig. 8). These regularities disappear when new behavioral rules emerge, as illustrated in Fig. 14. Clearly, there is a lot of autocorrelation structure in the time series of the evolutionary model when only optimists, pessimists, and two (and three) lag best responders are present in the population. However, as rules trying to exploit this structure enter the population these patterns disappear, as before (see the lower panels of Fig. 14). Our findings are thus very robust with respect to the specific updating mechanism used.

Our numerical analysis shows that introducing rules that try to exploit a particular time series structure indeed makes sure that cycles are arbitraged away but they do not necessarily stabilize the dynamics. In fact, adding rules typically complicates the dynamics and makes it more unpredictable. The reason for this is straightforward. Suppose the time series exhibits a $j$-cycle. If the ' $j-$ lag best responder' rule is available, then it will eventually attract most of the population of agents. However, given the negative feedback structure of the game, when a majority of agents uses this rule, it will lead to low payoffs. The negative feedback structure of the participation game therefore prevents the dynamics to lock in to a cycle of any period $j$ for which the corresponding $j-$ lag best responder rule is available in the heterogeneous agents model. Moreover our simulations have established that there exist values of the parameters such that the dynamics is chaotic and its autocorrelation structure is not significant at any order $j$ for which a best responder rule is used by agents. Best responders,

\footnotetext{
18 Judging from Fig. 12 there do exist values of $N$ and $\alpha$ where the dynamics converges to a 2-cycle. But how is this possible if we have established that when 2-lags BR are present no 2-cycle can appear? The solution to this apparent contradiction is that these are not 2 cycles but in fact 4-cycles where the dynamics of the participation rate has two values, a high one and a low one, in the sequence $\{$ high, high, low, low $\}$. Other than by the time series, not reported here, this is confirmed by the fact that when these 4-cycles appear the fraction of 2-lags BR is zero, as shown in the lower panels of Fig. 12, which is correct since 2 lags BR are always wrong along a 4-cycle.
} 

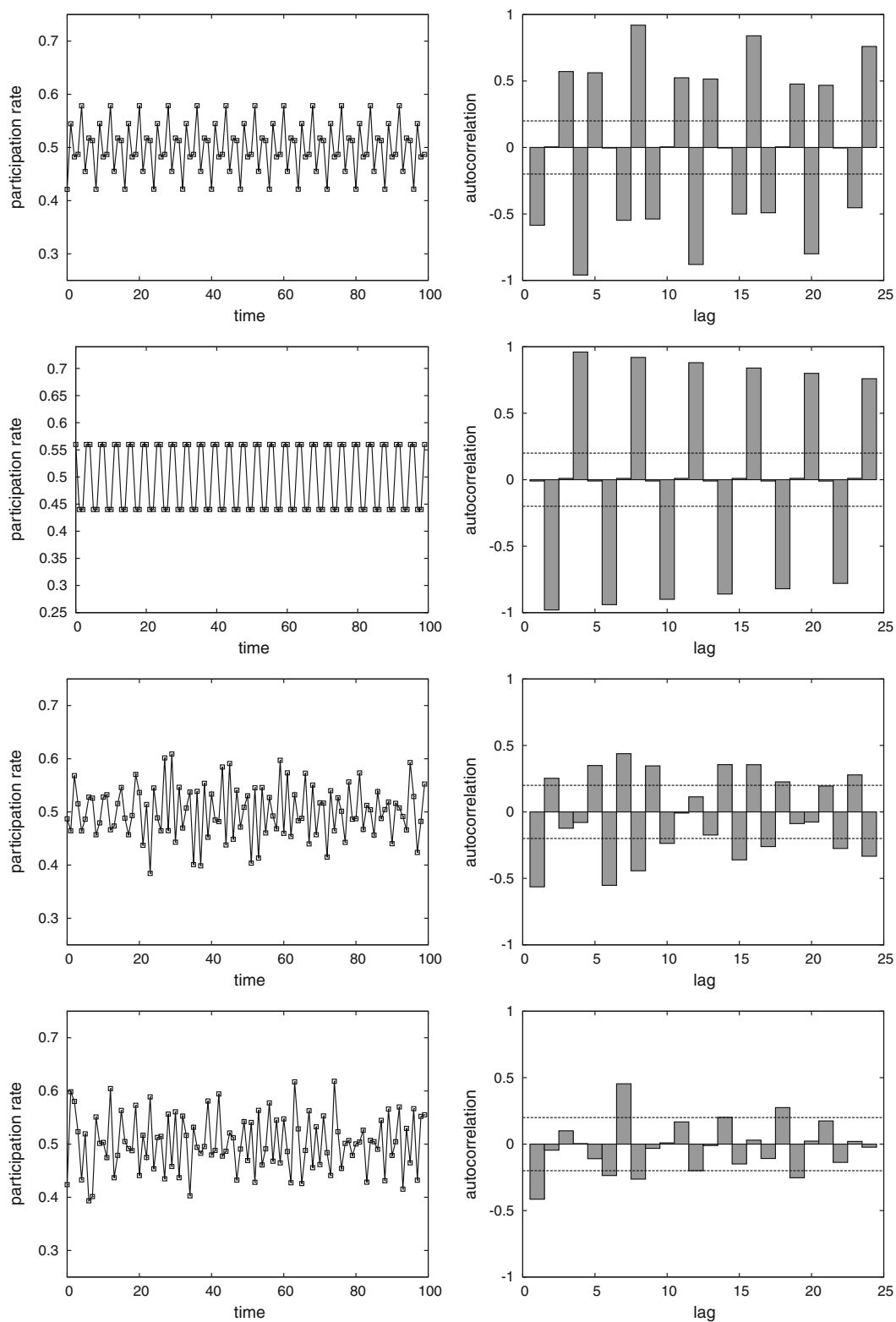

Fig. 14 Adjusted logit dynamics. Upper panels: participation rate and autocorrelation diagram for competition between optimists, pessimists, and 2-lag best responders. Middle-upper panels : participation rate and autocorrelation diagram for competition between optimists, pessimists, two, and three lag best responders. Middle-lower panels: participation rate and autocorrelation diagram for competition between optimists, pessimists, two, three, and four lag best responders. Lower panels: participation rate and autocorrelation diagram for competition between optimists, pessimists, and two to six lag best responders. The dotted lines show the 5\% significance levels of the autocorrelations. Autocorrelations and significance levels are based upon a sample of 100 observations. Parameters values are $N=300, b=\frac{1}{2}, \alpha=1, \beta=2 \gamma$ and $\gamma=\frac{1}{2}$ 
who survive out of exploiting the participation rate autocorrelation structure, have the effect of "arbitraging" these autocorrelations away.

\section{Conclusions}

Many economic decision problems can be characterized as negative feedback participation games. Experimental analyses of these games show that, although providing a reasonable description at an aggregate level, the symmetric mixed strategy Nash equilibrium does not explain individual behavior well. In particular, the participation rate is inherently unstable, and agents seem to use different rules to play the game. A series of contributions from computational economics, starting with the famous $E l$ Farol bar problem from Arthur (1994), also shows that complicated dynamics arise naturally in negative feedback participation games. In general, these models can not be investigated analytically and are difficult to study systematically, so that most results from this literature are based upon numerical simulations and, relatedly, are given for very specific choices of agents' rules and switching dynamics.

In this paper we have introduced an alternative model of evolutionary selection between behavioral rules, where each rule conditions its outcome on past participation rates. Our model is able to explain the experimental and computational results, but still is sufficiently simple to be analyzed theoretically, at least in its most stylized version, and general enough so that its results do not depend on the specific choice of behavioral rules and evolutionary dynamics.

For the simplest possible case of our model, where the only available two rules are those that specify to always participate, or to never participate, the participation rate dynamics evolves according to a nonlinear one-dimensional difference equation, which can be studied analytically. The symmetric MSNE is the only interior steady state of our evolutionary dynamics. We show that for a large number of players, a high speed of adjustment, or a high intensity of choice, overshooting occurs and the steady state becomes unstable. In that case, our simple model exhibits perpetual fluctuations in the participation rate around the symmetric MSNE.

The time series of participation rates has another feature: the time series exhibits certain regularities. When rules that try to exploit this structure are introduced, this particular structure disappears, but fluctuations around the symmetric MSNE do not vanish. Instead, other (higher order) regularities are introduced. Again, adding more sophisticated behavioral rules drives out these regularities, but does not stabilize the fluctuations, which are quite robust. This is also consistent with the literature. Arthur (1994), for example, finds that cycles are quickly arbitraged away in his computational model, by rules predicting those cycles. Relatedly, in a strategy experiment on the cobweb model, which has a structure that is similar to the negative feedback participation game, Sonnemans et al. (2004) find that strategies submitted by subjects become more complicated over time. However, the resulting price dynamics does not stabilize but becomes chaotic instead.

Acknowledgments We thank Cees Diks, Cars Hommes, Bill Sandholm, Yang Zhang and an anonymous referee for constructive comments and Peter Heemeijer for providing us with the experimental data used in Sect. 2. Opinions and errors remain ours. Pietro Dindo acknowledges financial support from the 
European Commission 6th FP (Contract CIT3-CT-2005-513396) Project: DIME - Dynamics of Institutions and Markets in Europe.

Open Access This article is distributed under the terms of the Creative Commons Attribution Noncommercial License which permits any noncommercial use, distribution, and reproduction in any medium, provided the original author(s) and source are credited.

\section{Appendix: Proofs of the Main Results}

Proof of Proposition 1 As argued in the text, the symmetric mixed strategy equilibrium $s^{*}$ corresponds to the solution Eq. 5 from Sect. 2.The function $p\left(x ; N_{c}, N\right)$ on the right-hand side of (5) is the cumulative distribution function (c.d.f.) of a binomial distribution, with $N-1$ degrees of freedom and probability of participating $x$, evaluated at $N_{c}-1$. This implies that for every $N_{c}<N$, it holds true that $p\left(0 ; N_{c}, N\right)=1$ and $p\left(1 ; N_{c}, N\right)=0$. Furthermore $p\left(x ; N_{c}, N\right)$ is continuous in $x$ and $\frac{\partial p\left(x ; N_{c} . N\right)}{\partial x}<0$ : if we increase the probability of participating the value of the c.d.f. at any fixed value between 0 and $N-1$ decreases. Consequently, since $\gamma<\beta, p\left(x ; N_{c}, N\right)=\frac{\gamma}{\beta}$ has a unique solution $s^{*}$ for any value of $N>1$, any $N_{c} \in[0, N-1]$ and any $\gamma<\beta$. Furthermore, since Eq. 5 does not depend on $\alpha$, neither does its solution $s^{*}$.

Typically $s^{*} \neq \frac{N_{c}}{N}$. We first show that when $N_{c}=\frac{1}{2} N$ and $\beta=2 \gamma$ then $s^{*}=$ $\frac{N_{c}}{N}=\frac{1}{2}$. Note that for all $N>1$ we have

$$
\begin{aligned}
1 & =\sum_{k=0}^{N-1}\left(\begin{array}{c}
N-1 \\
k
\end{array}\right)\left(\frac{1}{2}\right)^{k}\left(\frac{1}{2}\right)^{N-1-k} \\
& =\sum_{k=0}^{\frac{1}{2} N-1}\left(\begin{array}{c}
N-1 \\
k
\end{array}\right)\left(\frac{1}{2}\right)^{k}\left(\frac{1}{2}\right)^{N-1-k}+\sum_{k=\frac{1}{2} N}^{N-1}\left(\begin{array}{c}
N-1 \\
k
\end{array}\right)\left(\frac{1}{2}\right)^{k}\left(\frac{1}{2}\right)^{N-1-k} \\
& =p\left(\frac{1}{2} ; \frac{1}{2} N, N\right)+\sum_{j=N-1-k=0}^{\frac{1}{2} N-1}\left(\begin{array}{c}
N-1 \\
j
\end{array}\right)\left(\frac{1}{2}\right)^{N-1-j}\left(\frac{1}{2}\right)^{j} \\
& =p\left(\frac{1}{2} ; \frac{1}{2} N, N\right)+p\left(\frac{1}{2} ; \frac{1}{2} N, N\right)=2 p\left(\frac{1}{2} ; \frac{1}{2} N, N\right) .
\end{aligned}
$$

which gives $p\left(\frac{1}{2} ; \frac{1}{2} N, N\right)=\frac{1}{2}=\frac{\gamma}{\beta}$. Therefore $s^{*}=\frac{1}{2}$ is the unique MSNE for this specification.

In the final part of this proof we show that for general values of $\beta, \gamma<\beta, N$ and $b=\frac{N_{c}}{N} \in(0,1)$ we have $s^{*} \rightarrow b$ as $N \rightarrow \infty$. Define the random variable $\mathbf{n}=\frac{\mathbf{N}-\mathbf{1}}{N-1}$, where $\mathbf{N}-\mathbf{1}$ is a random variable with Bernoulli distribution with probability $s^{*}$ and $N-1$ degrees of freedom. Given the fact that $s^{*}$ solves Eq. (5), the $\gamma / \beta$ percentile of the distribution of $\mathbf{n}$ is given by $(b N-1) /(N-1)=b-(1-b) /(N-1)$. Also notice that the distribution of $\mathbf{n}$ has mean $s^{*}$ and variance $s^{*}\left(1-s^{*}\right) /(N-1)$. Note that when $N \rightarrow \infty$ the distribution of $\mathbf{n}$ is concentrated more and more around $s^{*}$. Assume there exists an $\varepsilon>0$ such that when $N \rightarrow \infty,\left|b-s^{*}\right| \geq \varepsilon$. This implies that 
either $s^{*}>b$ or $s^{*}<b$. If $s^{*}>b$, then $\operatorname{Pr}(\mathbf{n} \leq b-(1-b) /(N-1)) \rightarrow 0$ when $N \rightarrow \infty$. This contradicts that $s^{*}$ has been chosen such that the $\gamma / \beta$ percentile of $\mathbf{n}$ is $b-(1-b) /(N-1)$. On the other hand, if $s^{*}<b$, then when $N \rightarrow \infty, \operatorname{Pr}(\mathbf{n} \leq$ $b-(1-b) /(N-1)) \rightarrow 1$. This also contradicts that $s^{*}$ is such that the $\gamma / \beta$ percentile of $\mathbf{n}$ is given by $b-(1-b) /(N-1)$. We conclude that for every $\varepsilon>0,\left|b-s^{*}\right|<\varepsilon$ as $N \rightarrow \infty$.

Proof of Proposition 2 Given $K$ rules (6) and payoff functions (8) the evolutionary dynamics (9) can be expressed as a system of $2 K+L$ first order difference equations, where $L$ is the maximum number of lags used by any rule (i.e., $K$ equations for the behavioral rules, $p_{k, t}, K$ equations for the evolution of fractions, $x_{k, t}$, and $L$ equations to keep track of the lagged participation rates, $x_{t-l}$ ). A steady state is therefore a vector $\left(\mathbf{x}^{*}, \mathbf{p}^{*}, x^{*}, \ldots, x^{*}\right) \in \Delta^{K-1} \times\{0,1\}^{K} \times \mathbb{R}_{+}^{L}$, with $x^{*}=\mathbf{x}^{*} \cdot \mathbf{p}^{*}$. Since all lagged values are equal to each other at a steady state, the last $L$ equations do not impose any condition on the nature of a steady state. The $K$ values of $p_{k}^{*}$ are going to be either 0 or 1 and by property (13) we can therefore aggregate all rules with $p_{k}^{*}=1$ on the one hand, and all rules with $p_{k}^{*}=0$ on the other hand. As a result, the set of $K$ equations corresponding to (9-10) simplifies to one equation for the steady state aggregate participation rate, namely $x^{*}=x^{*}+\left(1-x^{*}\right) \rho_{01}-x^{*} \rho_{10}$, or

$$
\left(1-x^{*}\right) \rho_{01}=x^{*} \rho_{10} \text {. }
$$

Since by property (12) $\rho_{10}$ and $\rho_{01}$ can never be both positive, there can only be two types of solutions to (30). First, there exist boundary solutions that are characterized by either $x^{*}=0$ (and $\rho_{01}=0$ by property (14)) or by $x^{*}=1$ (and $\rho_{10}=0$ by property (14)). Many combinations of $\mathbf{x}^{*}$ lead to this type of steady state, among them all vectors with only one nonzero element, which is then equal to 1 . Second, there exist interior solutions with $0<x^{*}<1$ and therefore, by (30), $\rho_{01}=\rho_{10}=0$. By property (12) this implies that $\pi_{0}=\pi_{1}$, or equivalently, $\beta p\left(x^{*} ; N_{c}, N\right)=\gamma$, and therefore, by Proposition $1, x^{*}=s^{*}$.

To prove Propositions 3 and 4 the following result is useful.

Lemma 1 Let $\delta^{*}=\delta\left(s^{*} ; b, N\right)$. Then, for a fixed value of $b, \delta^{*}$ is decreasing in $N$ and $\delta^{*} \rightarrow-\infty$ as $N \rightarrow \infty$.

Proof The cumulative distribution of a binomial distribution with parameters $x$ and $N-1$ evaluated at $b N-1$ can be written in terms of the Beta-function, $B(x, y)=$ $\frac{(x-1) !(y-1) !}{(x+y-1) !}$, as

$$
p(x ; b N, N)=1-\frac{\int_{0}^{x} t^{N b-1}(1-t)^{N(1-b)-1} d t}{B(N b, N(1-b))}
$$

Equation (31) implies that we have

$$
\delta(x ; b, N)=\frac{\partial p(x ; b N, N)}{\partial x}=-\frac{x^{N b-1}(1-x)^{N(1-b)-1}}{B(N b, N(1-b))}
$$


The function $\delta(x ; b, N)$ has a unique maximum at $\widehat{x}_{b, N}=\frac{b N-1}{N-2}$. The associated minimum value of $\delta$ is given by get

$$
\begin{aligned}
\widehat{\delta}_{b, N} & =\delta\left(\widehat{x}_{b, N} ; b, N\right) \\
& =-\frac{(N-1) !}{(b N-1) !(N(1-b)-1) !}\left(\widehat{x}_{b, N}\right)^{b N-1}\left(1-\widehat{x}_{b, N}\right)^{N(1-b)-1} \\
& =-\frac{(N-1)(N-2) !}{(b N-1) !(N(1-b)-1) !}\left(\widehat{x}_{b, N}\right)^{b N-1}\left(1-\widehat{x}_{b, N}\right)^{N(1-b)-1}
\end{aligned}
$$

Taking the logarithm of $-\widehat{\delta}_{b, N}$ and applying the Stirling approximation formula, $\log (n !)=n \log (n)-n+\xi(n)$ where $\xi(n) \rightarrow 0$ as $n \rightarrow \infty$, we get

$$
\begin{aligned}
\log \left(-\widehat{\delta}_{b, N}\right)= & \log (N-1)+(N-2) \log (N-2)-(N-2) \\
& -(N b-1) \log \left(\frac{N b-1}{\widehat{x}_{b, N}}\right) \\
& -(N(1-b)-1) \log \left(\frac{N(1-b)-1}{1-\widehat{x}_{b, N}}\right)+(N-2)+\xi(N) \\
= & \log (N-1)+\xi(N)
\end{aligned}
$$

which goes to $\infty$ as $N \rightarrow \infty$ with the same speed as $\log (N)$ and therefore $\widehat{\delta}_{b, N} \rightarrow-\infty$ as $N \rightarrow \infty$. For the special case $b=\frac{1}{2}$ we have $\widehat{x}_{b, N}=\frac{1}{2}$ for all values of $N$ and, by Proposition $1, s^{*}=\frac{1}{2}$ for all even values of $N$. This implies that for $b=\frac{1}{2}$ we have $\delta^{*} \rightarrow-\infty$ as $N \rightarrow \infty$. For the general case with $b \neq \frac{1}{2}$, both $s^{*}$ and $\hat{x}_{b, N}$ converge to $b$ as $N$ goes to infinity. Moreover, $\delta(x ; b, N)$ is continuous in $x$. Consequently it holds $\delta^{*} \rightarrow-\infty$ as $N \rightarrow \infty$.

Proof of Proposition 3 and Corollary 1 The derivative of (22) is given by

$$
J(x)=1-\rho_{01}-\rho_{10}+(1-x) \frac{d \rho_{01}}{d x}-x \frac{d \rho_{10}}{d x},
$$

where, since $\pi_{0}$ is a constant, derivatives of the switching functions are

$$
\frac{d \rho_{01}}{d x}=\frac{\partial \rho_{01}}{\partial x}+\frac{\partial \rho_{01}}{\partial \pi_{1}} \frac{\partial \pi_{1}}{\partial x} \text { and } \frac{d \rho_{10}}{d x}=\frac{\partial \rho_{10}}{\partial x}+\frac{\partial \rho_{10}}{\partial \pi_{1}} \frac{\partial \pi_{1}}{\partial x} .
$$

Evaluating (32) at $x=0$, and using that $\left.\rho_{01}\right|_{x=0}=0$ by property (14), we obtain

$$
J(0)=\left.\left(1-\rho_{10}+\frac{d \rho_{01}}{d x}\right)\right|_{x=0} .
$$

Now because for $x=0$, due to property (12), we have $\pi_{1}>\pi_{0}$, we find that $\rho_{10}=0$. Moreover, since $\rho_{01}$ is constant for $x=0, \frac{\partial \rho_{01}}{\partial \pi_{1}}=0$. Consequently

$$
J(0)=1+\left.\frac{\partial \rho_{01}}{\partial x}\right|_{\left(x, \pi_{0}, \pi_{1}\right)=(0, \alpha, \alpha+\beta-\gamma)},
$$


which, due to (25), is always larger than one. Following the same reasoning for $x=1$, and using $\frac{\partial \rho_{10}}{\partial x}=-\frac{\partial \rho_{10}}{\partial x_{0}}$, we find

$$
J(1)=1-\left.\frac{\partial \rho_{01}}{\partial x}\right|_{\left(x, \pi_{0}, \pi_{1}\right)=(1, \alpha, \alpha-\gamma)}=1+\left.\frac{\partial \rho_{01}}{\partial x_{0}}\right|_{\left(x, \pi_{0}, \pi_{1}\right)=(1, \alpha, \alpha-\gamma)}
$$

Therefore, by (25), the steady state with $x=1$ is always unstable too.

Finally, let us evaluate (32) at $x=s^{*}$. Using that at $x=s^{*}$ payoffs are equal and thus $\rho_{01}=\rho_{10}=0$ by property 12 we obtain

$$
J\left(s^{*}\right)=\left.\left(1+\left(1-s^{*}\right) \frac{d \rho_{01}}{d x}-s^{*} \frac{d \rho_{10}}{d x}\right)\right|_{x=s^{*}} .
$$

Moreover, given that for $\pi_{0}=\pi_{1}$ we have $\rho_{01}=\rho_{10}=0$ for all $x$, we obtain $\frac{\partial \rho_{01}}{\partial x}=\frac{\partial \rho_{10}}{\partial x}=0$, and using that $\left.\frac{\partial \pi_{1}}{\partial x}\right|_{x=s^{*}}=\beta \delta^{*}$, we find that

$$
\left.\frac{d \rho_{j k}}{d x}\right|_{x=s^{*}}=\left.\beta \delta^{*} \frac{\partial \rho_{j k}}{\partial \pi_{1}}\right|_{x=s^{*}, \pi_{1}=\pi_{0}}
$$

for $j, k=0,1$ and $j \neq k$.

Because $\rho_{01}$ and $\rho_{10}$ as functions of $\pi_{1}$ have a kink at $\pi_{1}=\pi_{0}$ we have to distinguish between right and left derivatives. Using the fact that the right derivative of $\rho_{10}$ and the left derivative of $\rho_{01}$, both with respect to $\pi_{1}$, are zero, the right and left derivative of (32) evaluated in $x=s^{*}$ are

$$
J_{+}\left(s^{*}\right)=1+\left.\left(1-s^{*}\right) \beta \delta^{*} \frac{\partial \rho_{01}}{\partial_{+} \pi_{1}}\right|_{x=s^{*}, \pi_{1}=\pi_{0}},
$$

and

$$
J_{-}\left(s^{*}\right)=1-\left.s^{*} \beta \delta^{*} \frac{\partial \rho_{10}}{\partial_{-} \pi_{1}}\right|_{x=s^{*}, \pi_{1}=\pi_{0}},
$$

respectively.

Due to (26) both derivatives are always smaller than 1 so that $x=s^{*}$ is a locally stable steady state provided that both $J_{+}\left(s^{*}\right)>-1$ and $J_{-}\left(s^{*}\right)>-1$.

Using the expression above when the switching function takes the form (15) or (18) allows us to compute the derivatives for, respectively, the replicator dynamics and the adjusted logit dynamics. Straightforward computations show that, for both cases, $J_{-}\left(s^{*}\right)=J_{+}\left(s^{*}\right)$, and that the derivatives are given by (27) and (28) respectively.

Proof of Proposition 4 First observe that for any value of $\alpha$, any $\gamma>\beta$ and any $b \in(0,1)$ both the left and right derivatives go to $-\infty$ as $N \rightarrow \infty$. The latter is true since both derivatives are proportional to $\delta^{*}$ and from Lemma 1 it follows that 
$\delta^{*} \rightarrow-\infty$ as $N \rightarrow+\infty$. When $\beta=2 \gamma$ and $b=\frac{1}{2}$, we have $s^{*}=\frac{1}{2}$ (Proposition 1) for every $N$. As a result $\delta_{N}^{*}$ can be written as

$$
\delta_{N}^{*}=-\frac{\left(\frac{1}{2}\right)^{N-2}(N-1) !}{\left(\frac{1}{2} N-1\right) !\left(\frac{1}{2} N-1\right) !} .
$$

Note that $\delta_{2}^{*}=1$ and that for $N \geq 4$ we have $\delta_{N}^{*}=-\frac{3}{2} \times \frac{5}{4} \times \cdots \times \frac{N-1}{N-2}$. Note also that $\delta_{N}^{*}$ is monotonically decreasing in $N$ and that $\delta_{N}^{*} \rightarrow-\infty$ as $N \rightarrow \infty$. We then have that $J_{+}\left(s^{*}\right)$ and $J_{-}\left(s^{*}\right)$ only depend upon $N$ through $\delta_{N}^{*}$. Thus there exists an integer $M$ such that either $J_{+}\left(s^{*}\right)<-1$ or $J_{-}\left(s^{*}\right)<-1$, or both for $N>M$. This implies that $s^{*}$ is locally stable if and only if $N \leq M$.

\section{References}

Anderson, S. P., \& Engers, M. (2007). Participation games: Market entry, coordination, and the beautiful blonde. Journal of Economic Behavior and Organization, 63, 120-137.

Arthur, W. B. (1994). Inductive reasoning and bounded rationality. American Economic Review, 84, 406-411.

Bell, A. M. (2001). Reinforcement learning rules in a repeated game. Computational Economics, 18, 89-111.

Benaïm, M., \& Weibull, J. W. (2003). Deterministic approximation of stochastic evolution in games. Econometrica, 71, 873-903.

Bottazzi, G., \& Devetag, G. (2003). A laboratory experiment on the minority game. Physica A, 324, $124-132$.

Bottazzi, G., \& Devetag, G. (2007). Competition and coordination in experimental minority games. Journal of Evolutionary Economics, 17, 241-275.

Brianzoni, S., Cerqueti, R., \& Michetti, E. (2010). A dynamic stochastic model of asset pricing with heterogeneous beliefs. Computational Economics, 35, 165-188.

Brock, W. A., \& Hommes, C. H. (1997). Rational routes to randomness. Econometrica, 65, 1059-1095.

Brock, W. A., \& Hommes, C. H. (1998). Heterogeneous beliefs and routes to chaos in a simple asset pricing model. Journal of Economic Dynamics \& Control, 22, 451-481.

Brock, W. A., Dindo, P., \& Hommes, C. H. (2006). Adaptive rational equilibrium with forward looking agents. International Journal of Economic Theory, 2, 241-278.

Challet, D., \& Zhang, Y. C. (1997). Emergence of cooperation and organization in an evolutionary game. Physica A, 246, 407-418.

Challet, D., \& Zhang, Y. C. (1998). On the minority game: Analytical and numerical studies. Physica A, 246, 514-532.

Chiarella, C., \& He, X.-Z. (2002). Heterogeneous beliefs, risk and learning in a simple asset pricing model. Computational Economics, 19, 95-132.

Dindo, P. (2007). Bounded rationality and heterogeneity in economic dynamic models. PhD Thesis University of Amsterdam, Tinbergen Institute Research Series 396.

Droste, E., Hommes, C., \& Tuinstra, J. (2002). Endogenous fluctuations under evolutionary pressure in Cournot competition. Games and Economic Behavior, 40, 232-269.

Duffy, J., \& Hopkins, E. (2005). Learning, information and sorting in market entry games: Theory and evidence. Games and Economic Behavior, 51, 31-62.

Erev, I., \& Rapoport, A. (1998). Coordination, 'magic', and reinforcment learning in a market entry game. Games and Economic Behavior, 23, 146-175.

Franke, R. (2003). Reinforcement learning in the El Farol model. Journal of Economic Behavior \& Organization, $15,367-388$.

Fudenberg, D., \& Levine, D. K. (1998). The theory of learning in games. Cambridge: MIT Press.

Fudenberg, D., \& Tirole, J. (1991). Game theory. Cambridge: MIT Press.

Haltiwanger, J., \& Waldman, M. (1985). Rational expectations and the limits of rationalty: An analysis of heterogeneity. American Economic Review, 75, 326-340. 
Heemeijer, P. (2009). Expectation formation in dynamic market experiments. $\mathrm{PhD}$ Thesis University of Amsterdam, Tinbergen Institute Research Series 450.

Hofbauer, J., \& Sandholm, W. J. (2007). Evolution in games with randomly disturbed payoffs. Journal of Economic Theory, 132, 47-69.

Hofbauer, J., \& Sandholm, W. J. (2009). Stable games and their dynamics. Journal of Economic Theory, 144, 1665-1693.

Hommes, C. H. (1998). On the consistency of backward-looking expectations: The case of the cobweb. Journal of Economic Behavior \& Organization, 33, 333-362.

Iida, Y., Akiyama, T., \& Uchida, T. (1992). Experimental analysis of dynamic route choice behavior. Transportation Research B, 26, 17-32.

Lahkar, R., \& Sandholm, W. H. (2008). The projection dynamic and the geometry of population games. Games and Economic Behavior, 64, 565-590.

Li, T.-Y., \& Yorke, J. A. (1975). Period three implies chaos. American Mathematical Monthly, 82, 985-999.

Meyer, D. J., Huyck, J. B., Van Battalio, R. C., \& Saving, T. R. (1992). History's role in coordinating decentralized allocation decisions. Journal of Political Economy, 100, 292-316.

Neugart, M., \& Tuinstra, J. (2003). Endogenous fluctuations in the demand for education. Journal of Evolutionary Economics, 13, 29-51.

Rapoport, A., Seale, D., Erev, I., \& Sundali, J. (1998). Equilibrium play in large groups market entry games. Management Science, 44, 119-141.

Rota Bulò, S., \& Bomze, I. M. (2011). Infection and immunization: A new class of evolutionary game dynamics. Games and Economic Behavior, 71(1), 193-211.

Sandholm, W. H. (2003). Evolution and equilibrium under inexact information. Games and Economic Behavior, 44, 343-378.

Schlag, K. H. (1998). Why imitate, and if so, how? A boundedly rational approach to multi-armed bandits. Journal of Economic Theory, 78, 130-156.

Selten, R., Chmura, T., Pitz, T., Kube, S., \& Schreckenberg, M. (2007). Commuters route choice behavior. Games and Economic Behavior, 58, 394-406.

Sonnemans, J., Hommes, C., Tuinstra, J., \& van de Velden, H. (2004). The instability of a heterogeneous cobweb economy: A strategy experiment in expectation formation. Journal of Economic Behavior \& Organization, 54, 453-481.

Sundali, J. A., Rapoport, A., \& Seale, D. A. (1995). Coordination in market entry games with symmetric players. Organizational Behaviour and Human Decision Processes, 64, 203-218.

Taylor, P., \& Jonker, L. (1978). Evolutionary stable strategies and game dynamics. Mathematical Biosciences, 40, 145-156.

Weibull, J. W. (1995). Evolutionary game theory. Cambridge: MIT Press.

Zambrano, E. (2004). The interplay between analytics and computation in the study of congestion externalities: The case of the El Farol problem. Journal of Public Economic Theory, 6, 375-395.

Zwick, R., \& Rapoport, A. (2002). Tacit coordination in a decentralized market entry game with fixed capacity. Experimental Economics, 5, 253-272. 